\title{
Laser-Based Texturing of Graphene to Locally Tune Electrical Potential and Surface Chemistry
}

\author{
Manoj Tripathi, ${ }^{*}{ }^{\dagger}$ Alice King, ${ }^{\dagger}$ Giuseppe Fratta, ${ }^{\dagger}$ Manuela Meloni, ${ }^{\dagger}$ Matthew Large ${ }^{\dagger}$

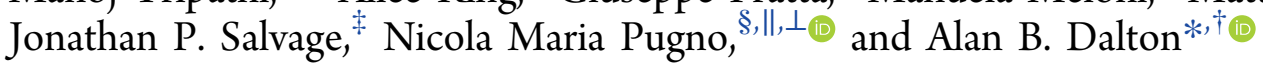 \\ ${ }^{\dagger}$ Department of Physics and Astronomy, University of Sussex, Brighton BN1 9RH, U.K. \\ ${ }^{\ddagger}$ School of Pharmacy and Biomolecular Science, University of Brighton, Brighton BN2 4GJ, U.K. \\ ${ }^{\S}$ Laboratory of Bio-Inspired \& Graphene Nanomechanics, Department of Civil, Environmental and Mechanical Engineering, \\ University of Trento, Via Mesiano 77, I-38123 Trento, Italy \\ "School of Engineering and Materials Science, Queen Mary University of London, Mile End Road, E1 4NS London, U.K. \\ ${ }^{\perp}$ Ket Lab, Edoardo Amaldi Foundation, Italian Space Agency, Via del Politecnico snc, I-00133 Roma, Italy
}

Supporting Information

\begin{abstract}
A simple procedure of producing three-dimensional blisters of graphene through irradiation of the visible range laser by Raman spectrometer has been presented. Fabrication of different volumes of the blisters and their characterization were carried out with Raman spectroscopy by tuning the irradiation dose. The produced blisters showed a consistency in altitude and a remarkable change in functionality, adhesion force map and local contact potential difference as compared to untreated monolayer graphene and naturally occurred graphene nanobubbles. Nevertheless, bilayer graphene is unaffected in the applied laser doses. The laser irradiation led to lattice expansion of carbon atoms and introduced oxygenic functional groups with the structural disorder. The internal pressure of the gaseous molecules was evaluated by monitoring the shape of the graphene blisters and nanobubbles. High-resolution Raman mapping showed the impact of laser-affected area and the defect density $\left(n_{\mathrm{d}}\right)$ is reported as a function of displacement. Our results reveal ease of applicability of the Raman laser for the imaging and texturing of graphene pointing toward the possibility of the desirable and cost-effective laser writing at the submicron scale by tuning photochemistry of graphene which is pivotal for numerous applications.
\end{abstract}

\section{INTRODUCTION}

The extraordinary properties of graphene have drawn a great deal of interest in the field of mechanics, ${ }^{1}$ electronics, ${ }^{2,3}$ photonics, ${ }^{4}$ and virtually all disciplines of science and technology. $^{5-7}$ The rapid progress in this emerging field resulted in tuning the extraordinary properties of graphene by altering the shape and dimensions, ${ }^{4,8}$ substrate interactions, ${ }^{3}$ and controlled functionalization., ${ }^{9,10}$ Inducing controlled and optimized strain in the graphene is another arising field known as "straintronics"11 to tailor the properties of graphene. Bending $^{12}$ and stretching ${ }^{4,13,14}$ of graphene through tensile force or mounting over textured surface ${ }^{15}$ are common practices to introduce a localized strain in two-dimensional (2D) materials. Nonuniform strain induces pseudomagnetic fields as high as $300 \mathrm{~T}$ and opens a bandgap in the electronic band structure. ${ }^{16,17}$ Biaxial strain in graphene can enhance the electron-phonon coupling and potentially turn graphene into a superconductor. ${ }^{18}$

Patterning and texturing of graphene through a laser (light amplification by stimulated emission of radiation) is one of the efficient ways for straining and altering electrical and mechanical properties. ${ }^{19-21}$ Controlled and refined laser treatment can induce subtle chemical and structural changes in graphene, which leads to tailoring bandgap, the lattice expansion, functionalization, and conductive channel formation. It can be produced by a variety of laser treatments such as continuous wave, ${ }^{22}$ nanosecond $d^{23-25}$ and femtosecond lasers. $^{26}$ Recently, complex and stable 3D structures of chemical vapor deposition graphene are produced by tuning the dose of irradiation to achieve desirable electrical and optical properties. $^{26,27}$ Nevertheless, it will be more efficient to produce 3D structure by a simple laboratory instrument using visible radiation. $^{21}$

Raman spectroscopy is considered as a sensitive and nondestructive laboratory tool for characterizing the chemical, mechanical, and electronic properties of graphene through vibrating carbon atoms. ${ }^{28-30}$ Raman spectroscopy is based on the vibrational transition of molecules occurring in the ground

Received: October 15, 2018

Accepted: November 29, 2018

Published: December 11, 2018 
(a)

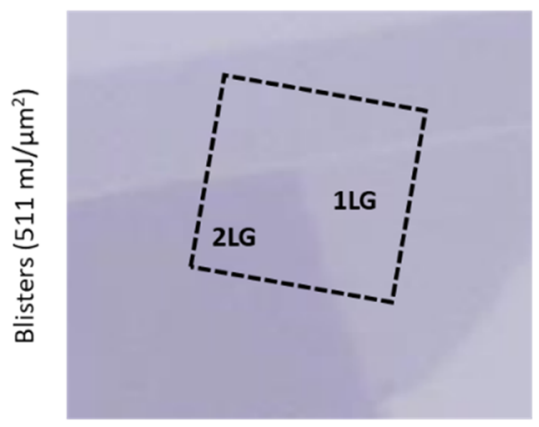

(d)

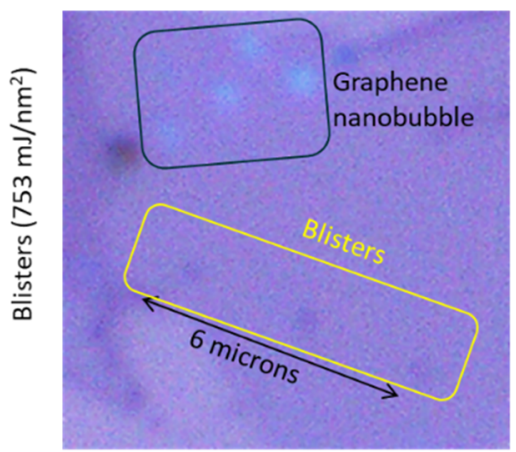

(b)

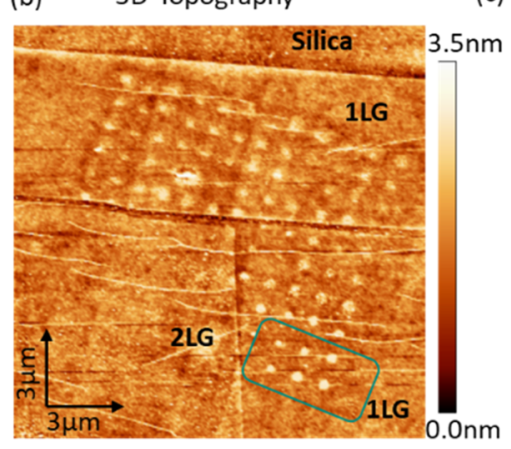

(e)

(c)

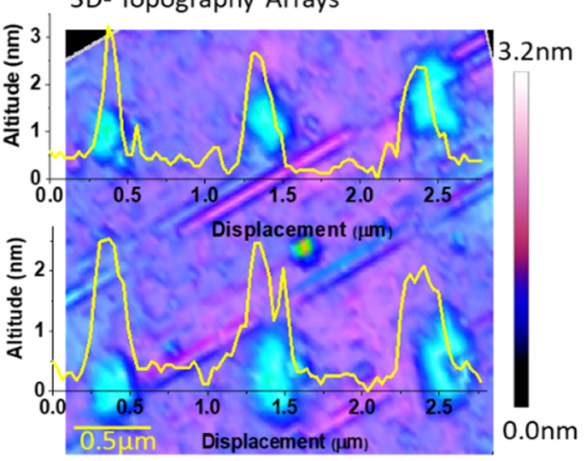

(f) 3D- Topography individual blister

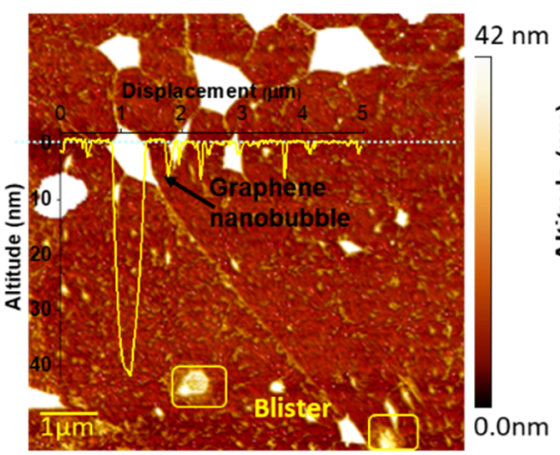

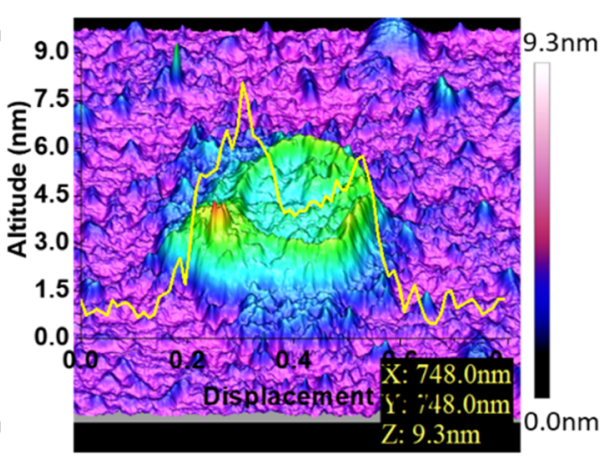

Figure 1. Area selection and blister formation. (a) Optical microscope image $(100 \times)$ of mechanically exfoliated graphene on silica before laser irradiation, with $1 \mathrm{LG}$ and $2 \mathrm{LG}$ graphene of area $20 \times 20 \mu \mathrm{m}^{2}$. The dotted square identifies the area of interest to characterize further the laserinduced 3D structure. (b) AFM topography is showing an array of graphene blisters only on $1 \mathrm{LG}$ at a power density of $511 \mathrm{~mJ} / \mu \mathrm{m}^{2}$. (c) Highresolution topography image of the blisters altitude up to $2.5 \pm 0.5 \mathrm{~nm}$, inset line profile shows consistency in the altitude of produced blisters. (d) Optical image of the 1LG (different from panel (a)) after Raman laser treatment $\left(753 \mathrm{~mJ} / \mu \mathrm{m}^{2}\right.$ ) produced three blisters separated by $2 \mu \mathrm{m}$ as marked by the yellow color rectangle. There are randomly generated graphene nanobubbles (referred as "Nb") near the edge of the $1 \mathrm{LG}$ marked by the black rectangle. (e, f) Three-dimensional (3D) topography of graphene nanobubble $40 \pm 2 \mathrm{~nm}$ and laser-induced blisters of altitude $6 \pm 1.5$ nm, respectively.

electronic state. The irradiation of the Raman beam in the visible range of light changes the lattice constant of the sample leading to variations in phonon frequencies. ${ }^{31}$ Nevertheless, the choice of laser is one of the primary concerns that is integrated into the Raman spectrometer system. Prolong exposure to laser excitation energies of $2.33 \mathrm{eV}(532 \mathrm{~nm})$ and $1.87 \mathrm{eV}(660 \mathrm{~nm})$ at the focusing area up to few microns can result in damage or modification in the sample. ${ }^{32}$ Lowmolecular-weight polymers are susceptible to initiate photochemistry/photophysics-induced morphology long before burning. The same applies to the adsorbed species in air conditions over catalysis. ${ }^{32,33}$ Nevertheless, tuned laser energy has been used to improve electrical properties in conjugated polymers by establishing planar conformations and higher conjugation length. ${ }^{34}$ Recently, a reversible cycle of $\mathrm{ClF}_{3}$ intercalated graphene blister engine is reported, which is ignited by $532 \mathrm{~nm}$ wavelength laser. The gaseous pressure generated up to $22.9 \mathrm{MPa}$ by $\mathrm{ClF}_{3}$ molecule under the irradiation expand the graphene into the $3 \mathrm{D}$ structure and return to the original position during shut-off laser, and the system acts as a sustainable piston. ${ }^{35}$ Continuous irradiation of Raman laser of $40 \mathrm{~mW}$ up to $2000 \mathrm{~s}$ can cause several stages of mechanical (blister) and chemical changes in the graphene/ silica surface useful to generate a desired pattern. ${ }^{21}$ Albeit a common laboratory instrument in material science, rare scientific evidences have been observed for the Raman laserinduced systematic modification of the graphene sample.
In this study, Raman lasers (532 and $660 \mathrm{~nm}$ ) at different power densities $\left(\mathrm{mJ} / \mu \mathrm{m}^{2}\right)$ have been irradiated over mechanically exfoliated graphene on silica. Atomic force microscopy (AFM) measures the maximum surface area affected under laser irradiation. A lower dose of power density $\left(2.5-3.7 \mathrm{~mJ} / \mu \mathrm{m}^{2}\right)$ has been used for analysis, whereas higher doses $\left(511-753 \mathrm{~mJ} / \mu \mathrm{m}^{2}\right)$ were used for the modifications. Different regions of single and bilayer graphene have been targeted in air condition. The 3D graphene structures produced showed distinguished morphology, adhesion force, and local surface potentials from untreated graphene crystal. The generation of $3 \mathrm{D}$ blisters is achieved through lattice expansion of carbon atoms and presence of trapped molecules. In addition, laser-induced partial oxidation of the graphene blisters has been verified from Raman spectroscopy. The areas affected by the laser irradiation were analyzed with Raman mapping as a function of displacement, and the defect density $\left(n_{\mathrm{d}}\right)$ was calculated. By tuning the power density of the laser treatment, desirable patterns of the altered electrical and mechanical properties are attainable. Thus, here, Raman spectrometer has been established as a detection tool which is utile for the modification of graphene.

\section{RESULTS AND DISCUSSION}

Morphology and Local Surface Potential of Blisters and Graphene Nanobubble. Raman lasers have been used for characterization (thickness, oxidation, strain) and fabrication of graphene $3 \mathrm{D}$ structure by changing the power density. 
(a)

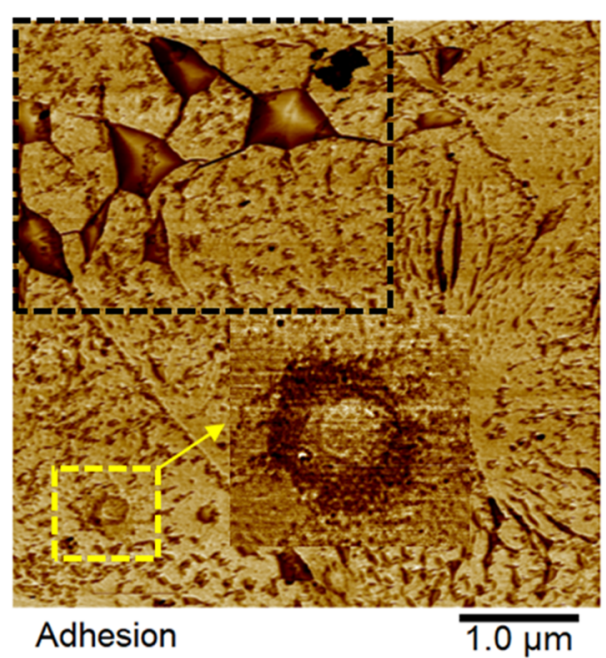

(b)

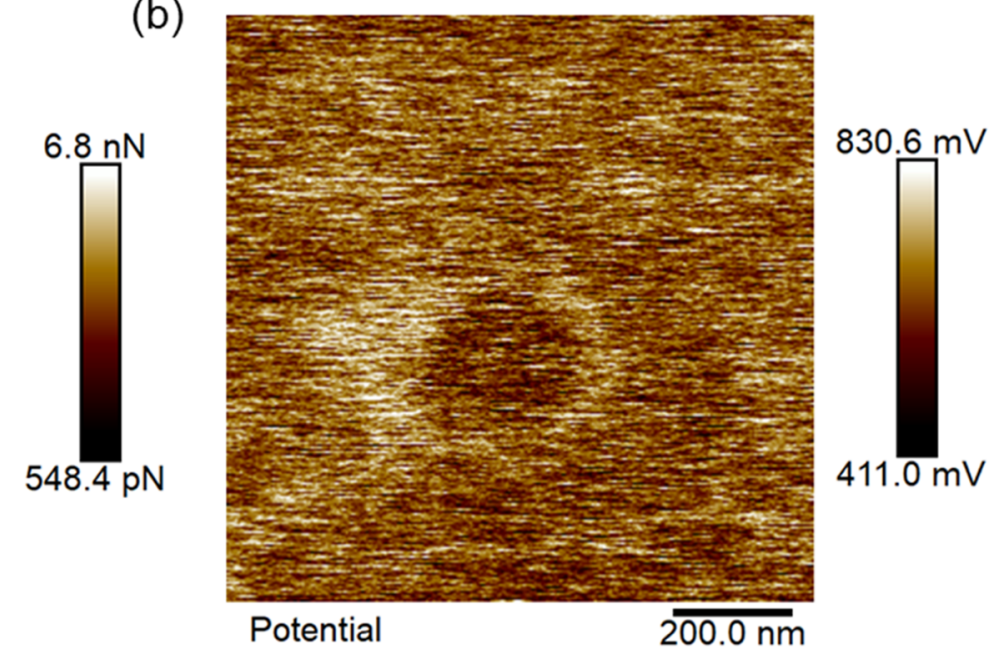

(c)

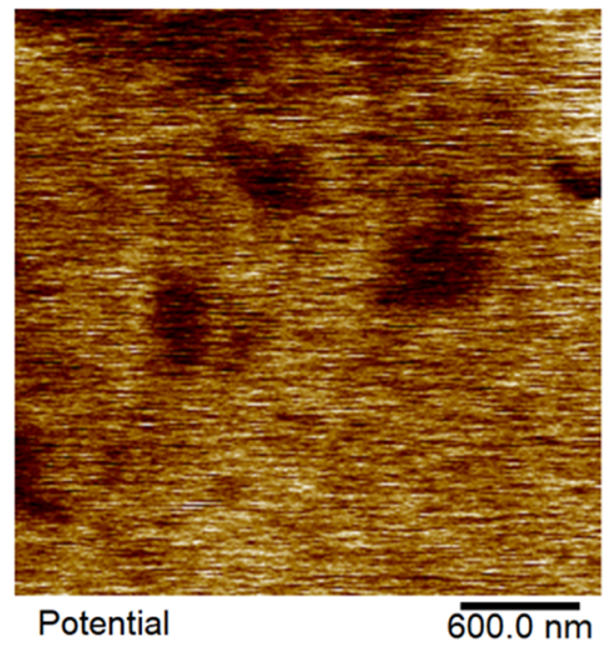

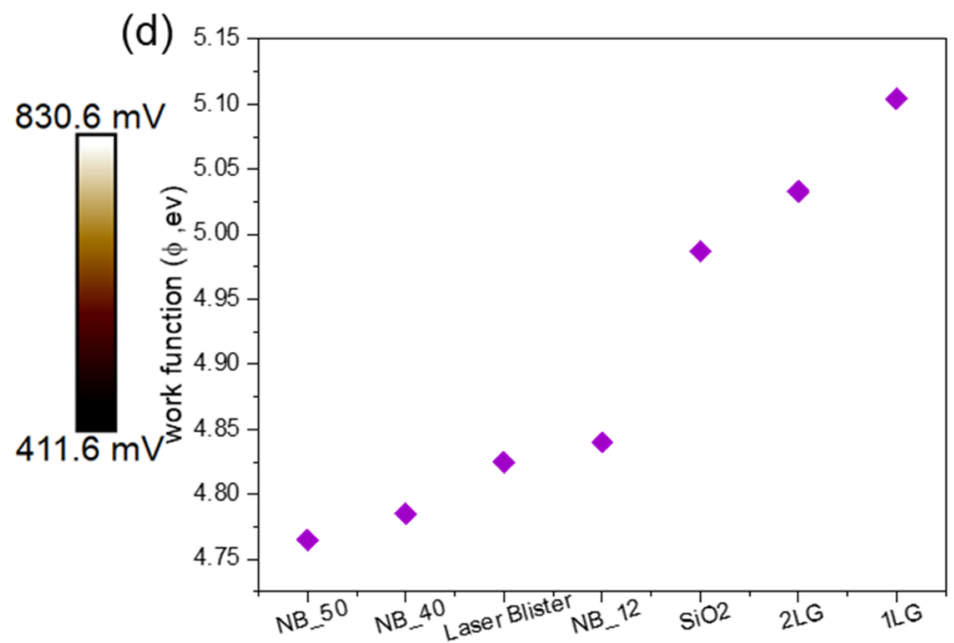

Figure 2. Adhesion force map and local surface potential characterization for graphene nanobubbles. (a) Adhesion force map (measured by pullout force) contrast distinguishes 1LG graphene into graphene nanobubble, blisters, ripple, and wrinkles. (b) The contact potential difference (CPD, $\mathrm{mV}$ ) value of an individual blister showing peculiar local surface potential with respect to 1LG and the nanobubbles (from panel c) by introducing a ring around inflated graphene with distinct CPD values. (c) The CPD map of the graphene nanobubbles varying with their altitude i.e., gap from the $\mathrm{SiO}_{2}$ substrate. The CPD values is highest for the basal plain graphene-supported silica. (d) The work function (eV) measured from the CPD for different regions shows highest value for $1 \mathrm{LG}$ graphene and lowest for the graphene nanobubble of altitude $50 \mathrm{~nm}$. The trend is showing that the substrate gap distance plays an important role in influencing local surface potential.

Lower power density $\left(2.55 \mathrm{~mJ} / \mu \mathrm{m}^{2}\right)$ was used for the thickness measurement at the initial stage (see Figure $S 1$ for details), whereas higher power densities (511 and $753 \mathrm{~mJ} /$ $\mu \mathrm{m}^{2}$ ) were implemented for the fabrication at different volumes. Mechanically exfoliated graphene was used as the test sample, which comprises single layer (1LG) and bilayer (2LG) over $300 \mathrm{~nm}$ oxide layer of silica on the silicon substrate, Figure 1a. The conferred region is crucial to investigate the significance of subsurfaces, i.e., a single layer of graphene beneath $2 \mathrm{LG}$ and silica substrate under 1LG. Under irradiation of power density $\left(511 \mathrm{~mJ} / \mu \mathrm{m}^{2}\right)$, arrays of $(12 \times 10)$ of $3 \mathrm{D}$ blisters of graphene are produced on $1 \mathrm{LG}$ only, nevertheless, 2LG was unaffected (Figure 1b). It depicts the vital role of interlayer coupling between the graphene layers in 2LG for distributing laser-induced heat energy by interlayer sliding. 1LG on silica substrate is resilient to slide due to higher shear strength ${ }^{36}$ and less capable of distributing the heat energy. The amorphous nature and lower thermal conductivity of silica $(1 \mathrm{~W} /(\mathrm{m} \mathrm{K}))$-trapped heat energy between graphene and silica interface cause further heating of
1LG with time (10-20 s), which then leads to a short-range distortion and buckling of the graphene layer. ${ }^{21,37}$

Controlled exposure of irradiation produces consistent altitude of graphene blisters of average height $2.5 \pm 0.5 \mathrm{~nm}$ for power density $511 \mathrm{~mJ} / \mu \mathrm{m}^{2}$ and up to $6 \pm 1.5 \mathrm{~nm}$ for 753 $\mathrm{mJ} / \mu \mathrm{m}^{2}$, Figures $1 \mathrm{c}-\mathrm{f}$ and S2. Higher altitude (up to $20 \mathrm{~nm}$ ) is reported in a controlled environment of argon and nitrogen gas by femtosecond laser. ${ }^{26}$ Nevertheless, continuous exposure of Raman laser at the high-power density in air condition at 33\% of relative humidity can bring chemical changes in the graphene that will be discussed in the subsequent sections.

The surface chemistry (i.e., functionalization) of the produced blisters is different from the naturally ensued graphene nanobubbles produced during preparation of the sample. The adhesion force map in Figure 2a distinguishes chemical structure of the laser-induced graphene blisters from the $1 \mathrm{LG}$ basal plain surface, wrinkles, ripples, and the graphene nanobubbles. The graphene nanobubbles are used as a reference $3 \mathrm{D}$ structure, which are produced by strain in the graphene, the presence of trapped molecules, and the 

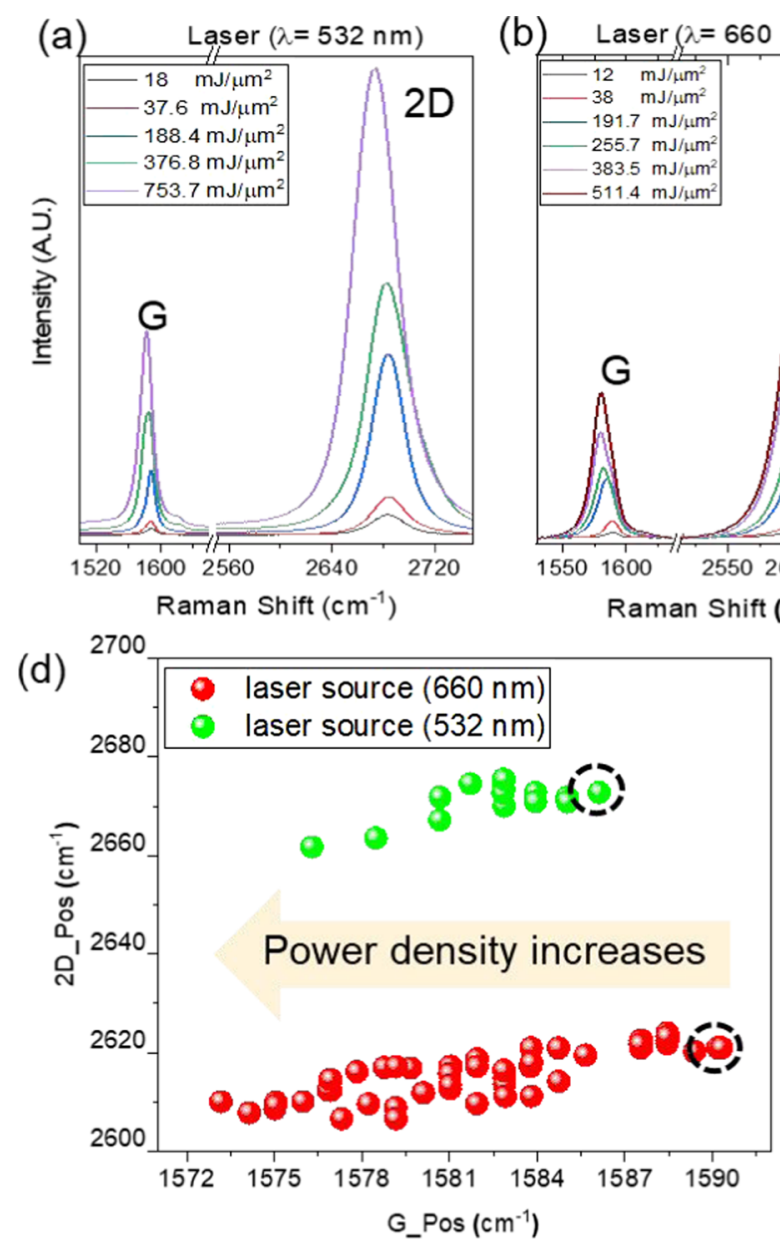
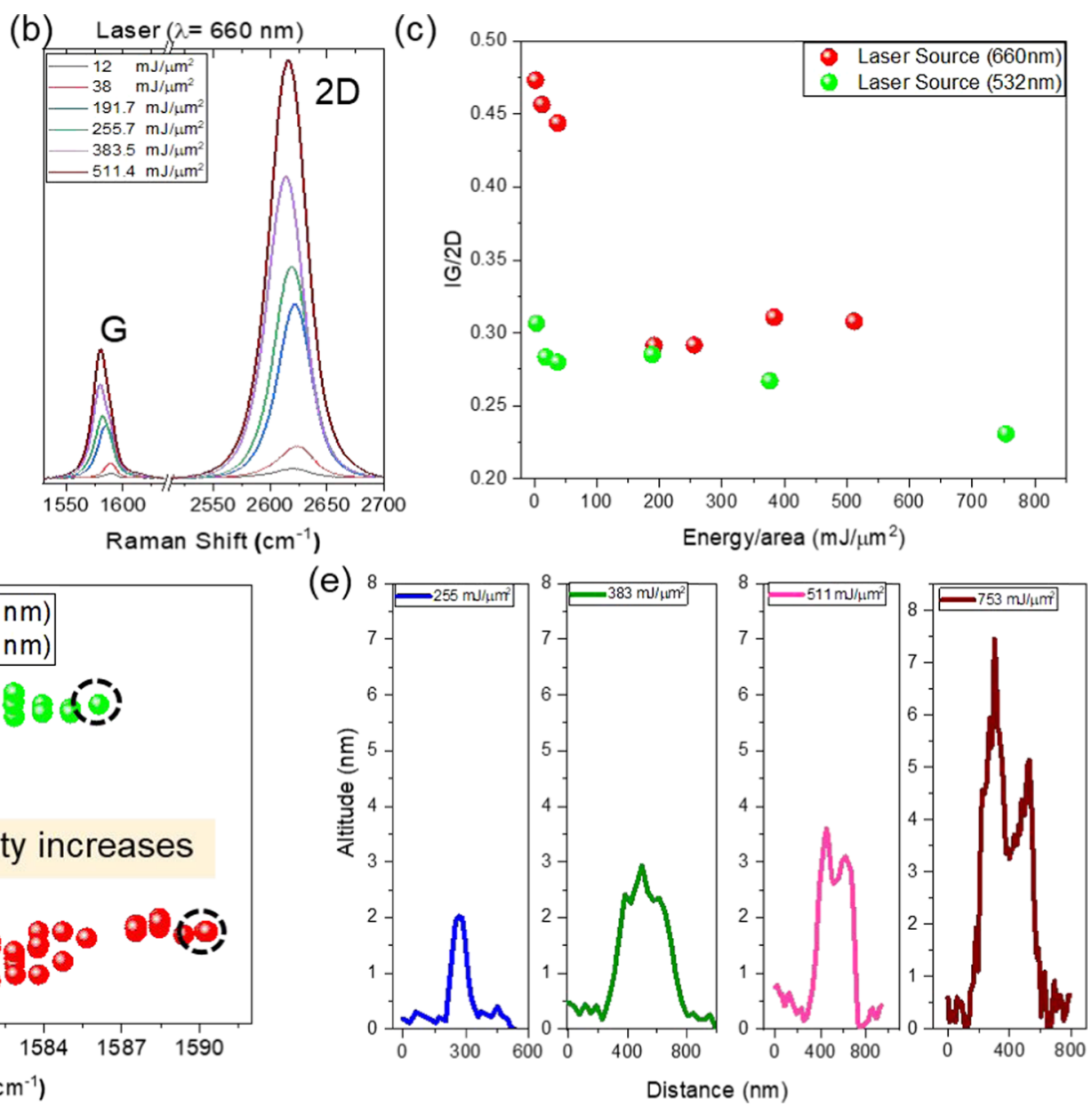

Figure 3. Stretching of graphene during irradiation. (a) Raman spectra of $1 \mathrm{LG}$ for lasers $\lambda=532 \mathrm{~nm}$ and (b) $\lambda=660 \mathrm{~nm}$ at different power densities are showing broadening of peaks $(\mathrm{G}$ and $2 \mathrm{D})$ and a red shift with increasing power density. (c) The sp ${ }^{2}$ domain of the graphene is systematically reduced for higher power density for both laser sources, as illustrated by decreasing intensity ratio $I_{\mathrm{G}} / I_{2 \mathrm{D}}$. (d) Red shift in $\mathrm{G}$ and $2 \mathrm{D}$ peak positions, the slopes produced by linear fit are $S_{\mathrm{g}}=1.9$ and $S_{\mathrm{r}}=1.1$ for different laser sources. Inset arrow is showing the direction of increasing power density. (e) AFM topographic profile of the blisters at different power densities showing an increase in volume.

deposition technique. These structural disorders are difficult to control during physical deposition of graphene but are useful to compare chemical and electrical characteristics from the laser-induced blisters. The adhesion force map illustrates the interaction between the silicon tip apex, elastic penetration, and the sample when the tip is moving vertically away from the surface, Figure S3. The adhesion force is linked with the contact area at the tip-graphene interface and the functional group attached on the graphene. Graphene nanobubble and the blisters resemble in adhesion force features, i.e., the adhesion force is higher at the central region of the structures than its surrounding curvature. It is due to the higher contact area between the tip apex and the central region of the blisters and at the graphene bubble. These central regions temporarily acted as deformed elastic membrane under normal force applied by tip apex. ${ }^{36}$ One can study the variation in adhesion force as a function of the graphene nanobubble volume. The present work is limited to comparison of properties between laser-induced and self-grown graphene structures.

Higher resolution (inset Figure 2a) of the adhesion force map of the blister illustrating a ring structure. It is in a contrast to the polygonic graphene nanobubble suggesting localized chemical perturbation in the blister. Local surface potential produced by KPFM also illustrates a ring structure of the blistered graphene of distinct contact potential difference (CPD), Figure $2 \mathrm{~b}$. Nearly $25-30 \%$ decrease is observed in the $\mathrm{CPD}$ values of the blistered central region compared to the untreated graphene. This resembles the wrinkled structure of graphene, which shows lower work function due to an increase of gap between graphene and silica substrates, which prohibits p-doing. ${ }^{27,38}$ The distinct CPD values between the inner and the outer ring of the blistered region also suggest oxidation of the graphene. ${ }^{21}$ Unlike blistered graphene, nanobubble CPD values are evenly distributed. The CPD values decrease with increasing altitude of the graphene nanobubble, Figure $2 c$, consequently the local work function $(\mathrm{eV})$. The trends of work function $(\mathrm{eV})$ for different regions are given at Figure $2 \mathrm{~d}$, which validates the influence of gap distance of graphene from the silica substrate. The work function of the 1LG measured as $5.02 \mathrm{eV}$ as the highest and $4.79 \mathrm{eV}$ for the $2 \mathrm{LG}$ at $33 \%$ of relative humidity are in coherence with the published findings. ${ }^{39,40}$ The lowering of work function for the $2 \mathrm{LG}$ can be explained by the screening of the charges (holes) originated from the $\mathrm{SiO}_{2}$ substrate by underlying graphene. ${ }^{39}$ Notably, the $\mathrm{CPD}$ values from the inner region of the ring were used for the work function measurement. This is in compliance with the CPD values measured for the nanobubble altitude up to 12 
$\mathrm{nm}$. These results decipher the locally tuned surface energy and electrical property for $1 \mathrm{LG}$.

Impact of Different Laser Power Density. The fabrication of blistered graphene can be achieved by controlling three major factors: (1) carbon-lattice expansion at $1 \mathrm{LG},{ }^{26,41}(2)$ presence of trapped molecules between $1 \mathrm{LG}$ and hydrophilic silica interface, ${ }^{35}$ and (3) oxidation of carbon atoms irradiated in air conditions. ${ }^{27}$ The expansion of carbon lattice was monitored by investigation of $\mathrm{G}$ and $2 \mathrm{D}$ peaks at different power densities by two different lasers $(\lambda=532$ and $660 \mathrm{~nm})$, Figure 3a,b. There is a broadening of G and 2D peaks with increasing power density for both lasers indicates increasing temperature. ${ }^{42}$ This shows the introduction of oxygenic functional group in air condition followed by structural disorder or creation of a localized region of holes doping by oxygen molecules, a similar situation observed by Liu et al. ${ }^{10}$ during thermal perturbation for the entire sample.

The frequencies of $\mathrm{G}$ and 2D peaks strongly depend on the change in charge density either by electrical, chemical, or thermal treatments owing to the static effect on bond lengths and nonadiabatic electron-phonon coupling. ${ }^{31,43}$ The G band $(\sim 1589)$ is associated with doubly generated (iTO and LO) phonon mode ( $E_{2 g}$ symmetry) at the Brillouin Zone center, which occurs due to first-order Raman scattering process in graphene. ${ }^{28}$ The $\mathrm{G}$ peak frequency is extremely sensitive to eventual changes in the oscillation strength of electronphonon interaction near the Fermi level. ${ }^{44}$ Generally, the phonon frequency of $\mathrm{G}$ band does not change by the energy of incident photon involved in Raman process. ${ }^{45}$ Nevertheless, prolonged exposure (up to $20 \mathrm{~s}$ ) of the lasers might cause chemical and structural changes in graphene due to heating, which can alter the Raman spectra. Therefore, dispersion of $G$ peak position with a variable irradiated power density $(\mathrm{mJ} /$ $\mu \mathrm{m}^{2}$ ) suggests a disorder in the carbon lattice.

The dispersion rate $\left(\Delta G_{\text {pos }} /\right.$ change in excitation laser) increases with disorder ${ }^{46}$ with the possible generation of local $\mathrm{sp}^{2}$ and $\mathrm{sp}^{3}$ domains in graphene. ${ }^{47}$ The excitation energy $\left(E_{\mathrm{L}}\right)$ leads to a raised bandgap $\left(E_{\mathrm{g}}\right)$ energy in graphene, which enhances the Raman scattering signal due to the resonance condition in the optical absorption. ${ }^{28,47}$ Several reports demonstrated the impact of laser for decreasing $\mathrm{sp}^{2} \mathrm{C}=\mathrm{C}$ bond and increment of $\mathrm{C}-\mathrm{C}$ bond. ${ }^{26,27} \mathrm{We}$ observed a decrease of $I_{\mathrm{G}} / I_{2 \mathrm{D}}$ ratio with higher power density, Figure $3 \mathrm{c}$ suggests an alteration in the hybrid state of $\mathrm{sp}^{2}$ carbon atoms up to $50 \%$ at the highest power density as compared to the unirradiated graphene.

Two-dimensional (2D) peak position shows upshift for the laser source $532 \mathrm{~nm}$ as compared to $660 \mathrm{~nm}$, Figure 3d. Unlike $\mathrm{G}$ frequency, the $2 \mathrm{D}$ band originates from a second-order process, involving two iTO phonons at the Dirac point $(K)$. The incident photon energy changes the phonon energy in the Raman process. As the photon energy increases, phonons farther from $K$ point are required for momentum conservation, which is responsible for significant dispersion. ${ }^{45,48}$ There is a softening of phonon modes due to lattice expansion for both lasers $(\lambda=532$ and $660 \mathrm{~nm})$ with a gradual increase of irradiation dose. Inset-marked frequencies for $2 \mathrm{D}$ - and $\mathrm{G}$ peak are measured at lowest power density from each laser. The slopes $(S)$ measured by the linear fit for each curve are $S_{\mathrm{g}}=1.9$ for $532 \mathrm{~nm}$ and $S_{\mathrm{r}}=1.1$ for $660 \mathrm{~nm}$ showing higher biaxial strain $^{49}$ for the former. Fairly large values of $S$ have been reported experimentally as $2.45,{ }^{14} 2.63^{13}$ and for theoretical prediction as $2.25^{50}$ for biaxially strained graphene over hollow silica substrate in the pressurized conditions. The expansion of the carbon lattice through Raman laser irradiation is significantly low as compared with pressurized blistered graphene. This is further confirmed by measuring the topographic profile of the blistered graphene at a different dose of the power density, Figure 3e. The maximum height achieved at highest power density $\left(753 \mathrm{~mJ} / \mu \mathrm{m}^{2}\right)$ is up to $6 \pm$ $1.5 \mathrm{~nm}$, which is less than pressurized blistered graphene. ${ }^{30,51}$

Role of Trapped Molecules between Graphene and Silica Interface. Graphene has shown impermeability to most of the gases, ${ }^{52}$ thus a differential pressure of several MPa can produce $3 \mathrm{D}$ structure. ${ }^{51}$ The role of trapped molecules to generate pressure and inflate graphene blister under laser irradiation was estimated by a geometrical approximation (Figure S4). The topographical informations (height $(h)$ and width $(w=2 r)$, where $r$ is its radius) were used to evaluate internal pressure $P$ of the graphene nanobubbles and the blisters by assuming hemispherical or cuboidal geometry. In particular, the force equilibrium imposes $P \pi r^{2}=2 \pi r t \sigma$ (hemispherical) or $P w^{2}=4 w t \sigma$ (cuboidal), where $t$ is the graphene thickness $(t=0.35 \mathrm{~nm})$ and $\sigma$ is its normal stress. Noting that $\sigma=E \varepsilon$, with $\varepsilon$ strain of the graphene and $E$ Young's modulus of graphene ( $E$ around $1 \mathrm{TPa})$ and that geometrically $\varepsilon=\frac{\Delta r}{r}=\frac{2 h}{w}$, we derive

$$
P=4 E t \varepsilon / w
$$

The geometry, stress, strain, and pressure inside the blisters or nanobubbles are reported in Table 1 ; the pressure is significantly lower in graphene blisters with respect to nanobubbles.

Table 1. Geometry, Strain, and Pressure Evaluations of the Graphene Blisters and Graphene Nanobubble ( $E=1 \mathrm{TPa}$; the Stress Could Be Estimated as $\sigma=E \varepsilon$ )

\begin{tabular}{|c|c|c|c|}
\hline width, $w(\mathrm{~nm})$ & height, $h(\mathrm{~nm})$ & strain, $\varepsilon$ & pressure, $P(\mathrm{MPa})$ \\
\hline \multicolumn{4}{|c|}{ Graphene blister } \\
\hline 156 & 1.87 & 0.024 & 215.15 \\
\hline 498 & 2.75 & 0.011 & 31.08 \\
\hline 484 & 3.40 & 0.014 & 40.64 \\
\hline 506 & 6.54 & 0.026 & 71.52 \\
\hline \multicolumn{4}{|c|}{ Graphene nanobubbles } \\
\hline 175 & 15 & 0.171 & 1371.43 \\
\hline 202 & 16 & 0.158 & 1097.93 \\
\hline 431 & 23 & 0.107 & 346.68 \\
\hline 542 & 46 & 0.170 & 438.45 \\
\hline 620 & 50 & 0.161 & 364.20 \\
\hline 646 & 40 & 0.124 & 268.38 \\
\hline 700 & 38 & 0.109 & 217.14 \\
\hline
\end{tabular}

The role of the trapped molecules between 1LG and silica interface is also evaluated by normally pressing graphene blistered at different normal forces from 0.250 to $10 \mathrm{nN}$. A systematic decrease in altitude of the blister was observed, see Figure S5. We successfully reduced the altitude of the blistered region up to $0.9 \pm 0.15 \mathrm{~nm}$ at $10 \mathrm{nN}$ of the normal force but were unable to regain its original configuration, which was 0.5 $\mathrm{nm}$ (measured by root mean square). The discrepancies in the values indicate the irreversibility of blistered graphene to regain its preceding conformation by normal pressing and also indicates the traces of the trapped molecules that did not escape completely. Separately, we did not observe any evident elevation of graphene blistered at the edge region due to 


\section{a Adhesion force map}

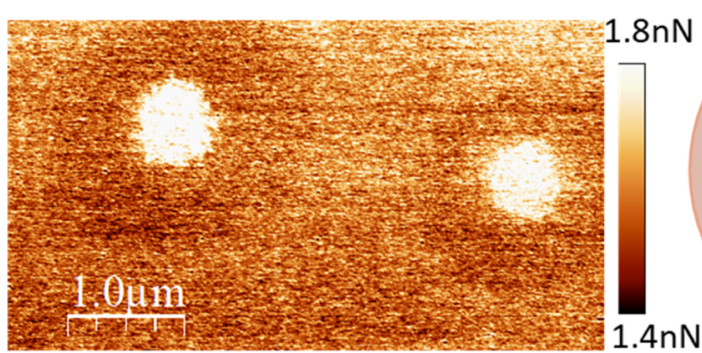

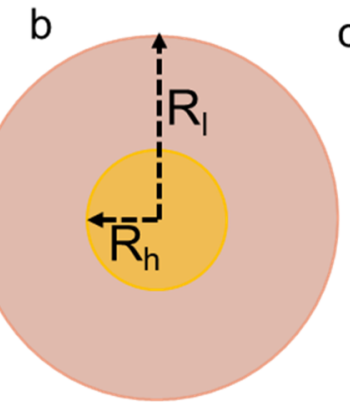

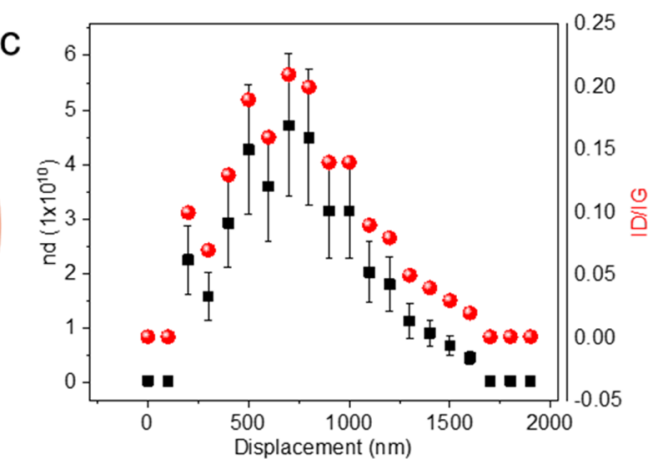

Figure 4. Affected area of the blister. Area affected by laser power density $\left(0.511 \mathrm{~mJ} / \mu \mathrm{m}^{2}\right)$ shown by (a) adhesion force map. The bright regions represent the higher values of the adhesion force between the tip apex and the surface. (b) Schematic image of the disordered region at two different radii $R_{\mathrm{h}}$ and $R_{\mathrm{i}}$ represent inner and outer circles, respectively. (c) The density of defects $\left(n_{\mathrm{d}}, 1 / \mathrm{cm}^{2}\right)$ and $I_{\mathrm{D}} / I_{\mathrm{G}}$ ratio as a function displacement.

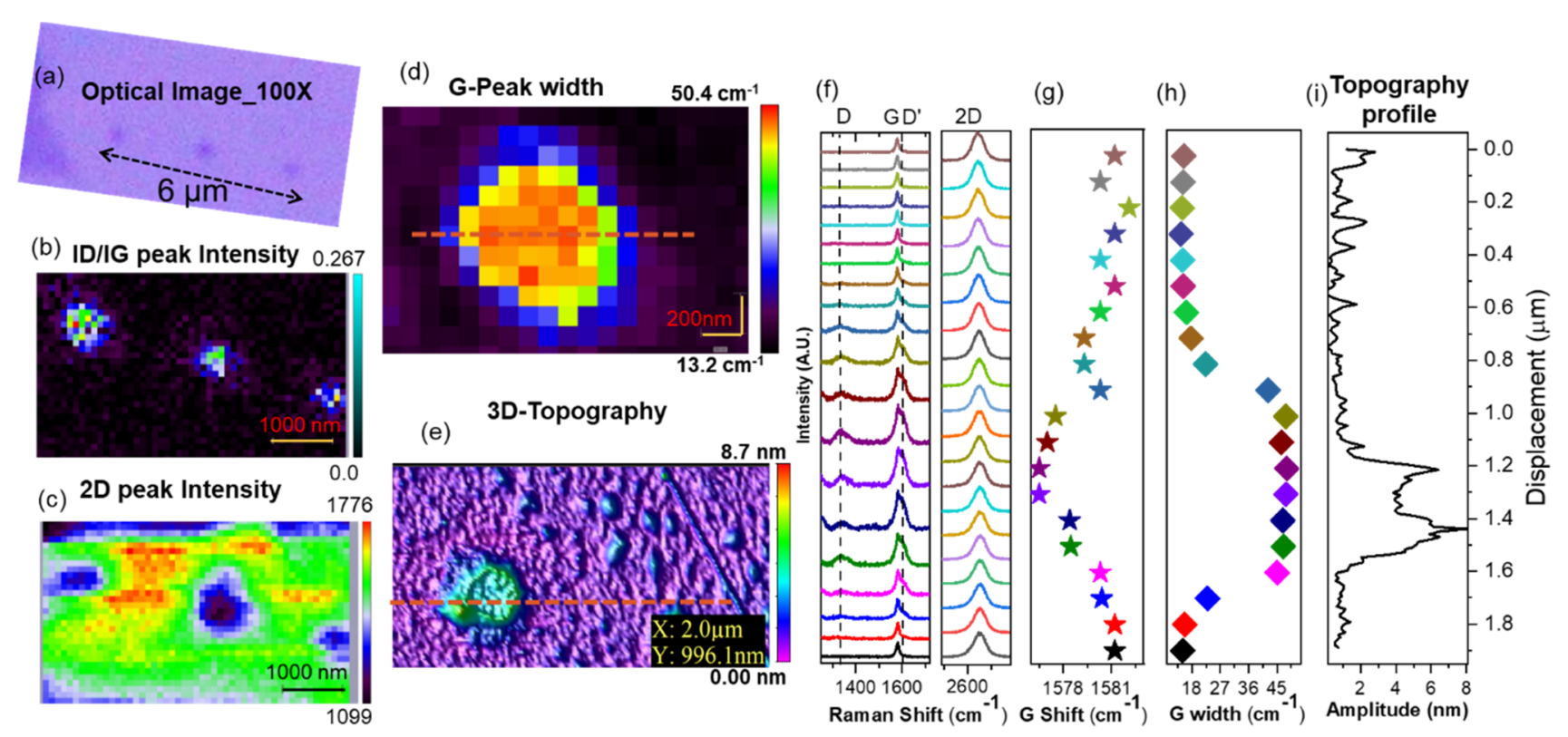

Figure 5. Correlation between topography and functionalization of graphene blister. (a) The optical microscopy of produced blisters (i.e., posttreated) at $100 \times$ optical lens. The separation between each blister is $2 \mu \mathrm{m}\left(\mathrm{b}-\mathrm{d}\right.$ ). Raman map (resolution $100 \mathrm{~nm}$, sampling data 1581 ) for $I_{\mathrm{D}} / I_{\mathrm{G}}$ ratio, $2 \mathrm{D}$ peak intensity, and $\mathrm{G}$ peak width in contrast to untreated graphene showing distinct signature from untreated single-layer graphene, scale bar is $200 \mathrm{~nm}$. (e) AFM 3D morphology of individual blister along with untreated surface. (f) Raman spectra of D- and G peak as a function of displacement showing a broadening of D and G peak at the blistered region. $(\mathrm{g}, \mathrm{h})$ There is broadening and red shift in $\mathrm{G}$ peak as a function of displacement. (i) The equivalent topography of the same blister shows maximum altitude of $6 \pm 1.5 \mathrm{~nm}$.

leakage of the trapped molecule ${ }^{35}$ although contrasting feature in the deformation map and adhesion force map has been observed indicating chemical perturbations (see Figure S6). Theoretical studies revealed that absence of the trapped molecule could also lead to the formation of blister up to $1 \mathrm{~nm}$ in altitude at a laser wavelength of $93.1 \mathrm{~nm}$, which might be based only on lattice extension. ${ }^{41}$

Extreme irradiation of the laser can lead to ablation of graphene carbon atoms and substrate underneath. ${ }^{53}$ Xing et al. observed graphene threshold damage limit $>300 \mathrm{GW} / \mathrm{cm}^{2}$ of power density, ${ }^{54}$ nevertheless disruption in $\mathrm{sp}^{2}$ carbon bond can be initiated at $14-66 \mathrm{~mJ} / \mathrm{cm}^{2}$. ${ }^{55}$ We did not observe any ablation of graphene and silica substrate in the given range of power density. Nevertheless, a wide area (diameter up to 1.8 $\mu \mathrm{m})$ of distorted graphene is observed by force-distance (F$D)$ spectroscopy through adhesion force map Figure 4a. The distorted area of graphene appeared in the annular fashion of radii $\left(R_{\mathrm{h}}\right)$ and $\left(R_{\mathrm{i}}\right)$, for $R_{\mathrm{h}}<R_{\mathrm{i}}$. The affected area associated with $R_{\mathrm{h}}$ is highly distorted regime as compared to $R_{\mathrm{l}}-R_{\mathrm{h}}$ region, where $R_{1}=3.3\left(R_{\mathrm{h}}\right)$. The produced deformation shapes resemble to the proposed model for the activated D-band scattering in the Raman spectrum of graphene sheet by the point-like defect. ${ }^{56}$ The model defines the generation of the circular area over a graphene of different radii during ions implantation. The inner area has a higher structural disordered region than the outer area where the Raman D-band is activated and lattice structure is preserved. We monitored the introduction of the point defects toward blistered center by analyzing the Raman spectra over the blistered region at low power density. A trend of $I_{\mathrm{D}} / I_{\mathrm{G}}$ ratio is observed, which has the higher values at the central region of the blister, Figure $4 \mathrm{c}$. The total area contributing to the $\mathrm{D}$ peak scattering is proportional to the number of point defects, giving rise to $I_{\mathrm{D}} / I_{\mathrm{G}}=A /\left(L_{\mathrm{d}}\right)^{2}$ where $A \approx 100 \mathrm{~nm}^{2}$ (for the low defect density) and $L_{\mathrm{d}}$ is the size of the crystalline $\mathrm{sp}^{2}$ clusters used to quantify the density of defects $\left(n_{\mathrm{D}}\right)$ from the following eq $2^{46,56}$ 


$$
n_{\mathrm{D}}\left(\mathrm{cm}^{-2}\right)=10^{14} /\left(\pi L_{\mathrm{D}}^{2}\right)
$$

Using finite values of $I_{\mathrm{D}} / I_{\mathrm{G}}$ at a fixed wavelength $\left(\lambda_{\mathrm{L}}\right)$, size of defect density $\left(n_{\mathrm{D}}\right)$ as a function of displacement is reported in Figure 4c. It clearly shows that the defect density decreases farther from the blistered region. The sizes of the defect density are in close proximity with simulation results showed for a height $\approx 1 \mathrm{~nm}$ produced by defect density $\sim\left(10^{9}\right.$ $\left.\mathrm{cm}^{-2}\right){ }^{26}$

Functionalization of Graphene. The impact of the laser to alter the local functionalization of $1 \mathrm{LG}$ is investigated as a function of displacement. Graphene blisters at Figure 5a are reinvestigated at a high-resolution physical AFM imaging (of $512 \times 512$ pixel $\approx 2 \mu \mathrm{m} \times 1 \mu \mathrm{m}$ ) and Raman mapping (pixel square $\approx 100 \mathrm{~nm} \times 100 \mathrm{~nm}$ ) at a lower dose laser energy for a fixed wavelength. The higher values of $I_{\mathrm{D}} / I_{\mathrm{G}}$ are localized at the blistered region as compared to its surrounding, Figure $5 \mathrm{~b}$ shows the introduction of Raman active disordered region. The decrease in the intensity of $2 \mathrm{D}$ peak (Figure 5c) and broadening of $\mathrm{G}$ peak width (Figure 5d) appeared at the central region of the blister. The correlation between blister's topography and Raman peaks was analyzed through line profile over the Raman mapped region, Figure 5d, (e.g., G peak width) and morphology at Figure 5e. The line profile over Raman map is associated to 20 Raman spectra of D, G, D', and $2 \mathrm{D}$ peaks taken at a separation of $100 \mathrm{~nm}$, Figure $5 \mathrm{f}$. It illustrates the orderly increment of the $\mathrm{D}$ peak intensity at $\sim 1335 \mathrm{~cm}^{-1}$, D' peak at $\sim 1609 \mathrm{~cm}^{-1}$ (high-resolution $\mathrm{D}^{\prime}$ peak is given at Figure S7). The 3D-topography of the given individual blister at Figure 5e is strongly linked to the red shift in the $\mathrm{G}$ peak up to $20 \mathrm{~cm}^{-1}$ (Figure $5 \mathrm{~g}$ ) and its broadening up to $50.4 \mathrm{~cm}^{-1}$ (Figure $5 \mathrm{~h}$ ). All these factors suggest a feature of partial oxidation with structural disorder, ${ }^{46,57}$ which is confirmed by the presence of the carboxylic group in Fourier transform infrared (FT-IR) spectra at Figure S8. These findings were also observed by Johansson et al. ${ }^{26}$ to induce epoxy and hydroxyl groups after irradiation in air conditions.

Raman laser writing can produce an array of 3D graphene in different designs, periodicity, or a complex script. Here, "US" depicts an acronym for University of Sussex with tuned local CPD potential and adhesion force map at Figure S9. The method illustrated here opens new avenues and possibilities in the graphene-based devices to generate localized pseudomagnetic field, ${ }^{16}$ sustainable and efficient nanoscale motor ${ }^{35}$ with different intercalated molecules, localized functionalization surface of different adhesion forces, and polarities for biosensing applications, ${ }^{58}$ bandgap tailoring, ${ }^{59}$ nanofluid, and actuator. ${ }^{41}$ Here, we proposed an effective and simple method to produce $3 \mathrm{D}$ structure of graphene with tuned surface properties.

\section{CONCLUSIONS}

We demonstrated a procedure of producing the localized 3D structure of single-layer graphene by tuning laser dose from Raman spectrometer. Increase in the dose of power density from 511 to $753 \mathrm{~mJ} / \mu \mathrm{m}^{2}$ surges the volume of the blister. The lattice expansion of carbon atoms increases the gap between ILG and silica interface and deposition of the oxygenic functional group, which alters the photoinduced chemistry of graphene and is outwardly shown through scanning probe techniques (AFM and KPFM). The introduction of the functional group and higher contact area of deformation causes the higher force of adhesion at the blistered region. The CPD contrast showed the lower work function at the blistered region due to the prohibition in p-doping from the silica substrate, which resembles the wrinkle and graphene nanobubble characteristics. The work function decreases with increasing gap between graphene and the silica substrate. The internal pressures in the blisters are significantly lower than the graphene nanobubble. We did not observe any laser-induced ablation of graphene or any vacancy in the irradiated region, though the structural disorder was received. The decrease in intensity $\left(I_{\mathrm{G}} / I_{2 \mathrm{D}}\right)$ with increasing power density shows a reduction in the domain size of the $\mathrm{sp}^{2}$ breathing mode. Phonon softening observed in high-resolution Raman mapping toward the central part of the blistered region shows lattice expansion of the carbon atoms. The defect density measured from $I_{\mathrm{D}} / I_{\mathrm{G}}$ decreases as it goes away from the laser-affected region $\left(R_{\mathrm{h}}\right.$ to $\left.R_{\mathrm{l}}\right)$, affirmed an annular shape for the distribution of defects. Our results reveal the effectiveness of the Raman spectrometer for the modifications and functional analysis of graphene layer.

\section{MATERIALS AND METHOD}

Single and bilayer graphene was produced by mechanical exfoliation of graphite (HOPG) and deposited on a silica substrate (300 nm oxide thickness). Deposited graphene on silica substrate was cleaned in an ultrasonic bath in the sequence of isopropanol and deionised water each for $30 \mathrm{~min}$, then heat-treated in vacuum for $3 \mathrm{~h}$ at $200{ }^{\circ} \mathrm{C}$.

Raman spectroscopy (spectral resolution $0.3 \mathrm{~cm}^{-1}$ ) has been carried out at $100 \times$ objective lens using two lasers. Laser (1): $532 \mathrm{~nm}$ (type: solid state, model: RL53250) and Laser (2): $660 \mathrm{~nm}$ (type solid state, model: RL660C100) at different powers $(\mathrm{mW})$. The maximum power used from the laser source for $532 \mathrm{~nm}$ is $50 \mathrm{~mW}$ and for $660 \mathrm{~nm}$ is $100 \mathrm{~mW}$. Variations in the laser powers were carried through ND filters. The higher power densities of the laser were achieved by increasing the time duration $(10,15$, and $20 \mathrm{~s})$ of the irradiation while keeping the output power consistent. It should be noted that there is a loss of $5-10 \%$ of the power density during reflection from in-built mirrors in the Raman spectrometer. The laser irradiation was repeated over four different single-layer graphene flakes of reproducible textured amplitudes, which are reported at Figures 1, 5, S5, S6, and S8.

AFM characterization was performed with a Bruker Dimension Icon, positioned in an insulated box over an antivibrant stage to minimize environmental noise and building vibrations. Contact potential difference (CPD, volts) and mechanical data were measured from advanced operation mode of PF-KPFM (PeakForce-Kelvin Probe Force Microscopy) and PF-QNM (PeakForce-Quantitative nanomechanical), respectively. PeakForce is a Bruker's proprietary mode, which allows the collection of both types of information on a single acquisition. Amid the PF-KPFM operation, the standard PeakForce procedure during the first pass of each line scanning, with the tip softly $(<1 \mathrm{nN})$ tapping on top of the sample. In this condition, it gathers topography and mechanical properties of the sample. In the second pass over the same scanning line, the cantilever lifted from the surface up to $10 \mathrm{~nm}$ distance to collect CPD data. The proprietary Scanasyst algorithms simplify engaging and parameters for setting procedures, optimizing in real time Scan Rate, PeakForce set point and Feedback Gain settings but was in general turned off during capture once the optimal parameters were achieved to avoid inconsistency forth the final picture. 
This type of characterization has been performed using every time the same mounted tip to guarantee as much consistency as possible in the Kelvin Probe data, highly variable, being dependent not only on sample properties but also on the cantilever used in the measurement. A PFQNE-AL tip was chosen for these reasons, being the gold standard of Bruker's tip for KPFM characterization. It is a soft silicon-nitride tip with $5 \mathrm{~nm}$ nominal tip diameter, $300 \pm 100 \mathrm{kHz}$ resonant frequency, and $0.8 \pm 0.2 \mathrm{~N} / \mathrm{m}$ spring constant, optimized for electrical modes and with a proprietary reflective coating on the backside. Thermal Tune calibration was performed before each imaging session to verify consistency of resonance frequency and stiffness of the cantilever. The stiffer cantilever has been avoided to minimize the physical damage to the blister.

High-resolution mechanical information acquired by PeakForce-QNM (Quantitative Nanomechanical Mapping) for Figure 2a, S3, S5, and S6. This mode works with the same procedure described in the first pass of the PF-KPFM. A silicon nitride (model: Scanasyst-air tip) was chosen in these cases, having a nominal ultrasharp tip of $2-3 \mathrm{~nm}$ that allows maximum resolution while minimizing contact area, ensuring better consistency and resolution of mechanical information, in particular adhesion force map. The resonance frequency and the stiffness of the cantilever are measured as $70 \pm 25 \mathrm{kHz}$ and $0.2-0.8 \mathrm{~N} / \mathrm{m}$ (nominal $0.4 \mathrm{~N} / \mathrm{m}$ ), respectively.

The adhesion force measurement carried out in PF-QNM approach was operated in a true contact mode at relative humidity $(30-35 \%)$ at room temperature, the blisters were bearing a certain contact load and may have a larger contact deformation. $^{60}$ Thus, the elastic deformation is performed by modest pressing (set point $<1 \mathrm{nN}$ ) over the blistered region. It is a distance between "jump to contact" and the elastic penetration. Prominent deformation at the blistered membrane leads to the higher contact area between the AFM tip-apex and the deformed region, which is responsible for higher adhesion forces "pull-out". 36

The FT-IR measurements were performed using PerkinElmer Spotlight 400 FT-IR Microscope System. The system uses dual-mode single point and mercury cadmium telluride array detector standard with InGaAs array option for optimized NIR imaging. All the measurements were done using the mid-IR $\left(4000-500 \mathrm{~cm}^{-1}\right)$.

\section{ASSOCIATED CONTENT}

\section{S Supporting Information}

The Supporting Information is available free of charge on the ACS Publications website at DOI: 10.1021/acsomega.8b02815.

Thickness verification of graphene layers and absence of $D$ peak confirmed by Raman spectroscopy; optical image of blister produced at different locations in 1LG; topography of individual blister with adhesion and deformation map produced at a laser dose of $511 \mathrm{~mJ} /$ $\mu \mathrm{m}^{2}$; inducing normal force on blisters at different set points with peakforce schematic mechanisms; impact of laser at the edge region of graphene; red shift and broadening of $\mathrm{G}$ and $2 \mathrm{D}$ peaks with increasing laser dose (PDF)

\section{AUTHOR INFORMATION}

\section{Corresponding Authors}

*E-mail: m.tripathi@sussex.ac.uk (M.T.).

*E-mail: a.b.dalton@sussex.ac.uk (A.B.D.).

ORCID

Nicola Maria Pugno: 0000-0003-2136-2396

Alan B. Dalton: 0000-0001-8043-1377

Notes

The authors declare no competing financial interest.

\section{ACKNOWLEDGMENTS}

M.T. is supported by University of Sussex strategic development fund. G.F. and M.M. are thankful to European Union's Horizon 2020 research and innovation program under the Marie Sklodowska-Curie grant agreement (642742). N.P. is supported by the European Commission H2020 under the Graphene Flagship Core 2 No. 785219 (WP14 “Composites”) and FET Proactive "Neurofibres" Grant No. 732344 as well as by the Italian Ministry of Education, University and Research (MIUR) under the "Departments of Excellence" grant L.232/ 2016.

\section{REFERENCES}

(1) Lee, C.; Wei, X.; Kysar, J. W.; Hone, J. Measurement of the elastic properties and intrinsic strength of monolayer graphene. Science 2008, 321, 385-388.

(2) Geim, A. K. Graphene: status and prospects. Science 2009, 324, $1530-1534$

(3) Zhou, S. Y.; Gweon, G.-H.; Fedorov, A.; First, P.; De Heer, W.; Lee, D.-H.; Guinea, F.; Neto, A. C.; Lanzara, A. Substrate-induced bandgap opening in epitaxial graphene. Nat. Mater. 2007, 6, No. 770.

(4) Stöhr, R. J.; Kolesov, R.; Xia, K.; Wrachtrup, J. All-optical highresolution nanopatterning and $3 \mathrm{D}$ suspending of graphene. ACS Nano 2011, 5, 5141-5150.

(5) Geim, A. K.; Novoselov, K. S. The rise of graphene. Nat. Mater. 2007, 6, 183.

(6) Geim, A. K.; Kim, P. Carbon wonderland. Sci. Am. 2008, 298, 90-97.

(7) Novoselov, K. S.; Fal, V.; Colombo, L.; Gellert, P.; Schwab, M.; Kim, K. A roadmap for graphene. Nature 2012, 490, 192.

(8) Barone, V.; Hod, O.; Scuseria, G. E. Electronic structure and stability of semiconducting graphene nanoribbons. Nano Lett. 2006, 6, $2748-2754$.

(9) Elias, D. C.; Nair, R. R.; Mohiuddin, T.; Morozov, S.; Blake, P.; Halsall, M.; Ferrari, A.; Boukhvalov, D.; Katsnelson, M.; Geim, A.; et al. Control of graphene's properties by reversible hydrogenation: evidence for graphane. Science 2009, 323, 610-613.

(10) Liu, L.; Ryu, S.; Tomasik, M. R.; Stolyarova, E.; Jung, N.; Hybertsen, M. S.; Steigerwald, M. L.; Brus, L. E.; Flynn, G. W. Graphene oxidation: thickness-dependent etching and strong chemical doping. Nano Lett. 2008, 8, 1965-1970.

(11) Si, C.; Sun, Z.; Liu, F. Strain engineering of graphene: a review. Nanoscale 2016, 8, 3207-3217.

(12) Zhang, D.-B.; Akatyeva, E.; Dumitrică, T. Bending ultrathin graphene at the margins of continuum mechanics. Phys. Rev. Lett. 2011, 106, No. 255503.

(13) Metzger, C.; Rémi, S.; Liu, M.; Kusminskiy, S. V.; Castro Neto, A. H.; Swan, A. K.; Goldberg, B. B. Biaxial strain in graphene adhered to shallow depressions. Nano Lett. 2009, 10, 6-10.

(14) Zabel, J.; Nair, R. R.; Ott, A.; Georgiou, T.; Geim, A. K.; Novoselov, K. S.; Casiraghi, C. Raman spectroscopy of graphene and bilayer under biaxial strain: bubbles and balloons. Nano Lett. 2012, 12, 617-621.

(15) Gill, S. T.; Hinnefeld, J. H.; Zhu, S.; Swanson, W. J.; Li, T.; Mason, N. Mechanical control of graphene on engineered pyramidal strain arrays. ACS Nano 2015, 9, 5799-5806. 
(16) Levy, N.; Burke, S.; Meaker, K.; Panlasigui, M.; Zettl, A.; Guinea, F.; Neto, A. C.; Crommie, M. Strain-induced pseudomagnetic fields greater than 300 tesla in graphene nanobubbles. Science 2010, 329, 544-547.

(17) Guinea, F.; Katsnelson, M.; Geim, A. Energy gaps and a zerofield quantum Hall effect in graphene by strain engineering. Nat. Phys. 2010, 6, 30.

(18) Si, C.; Liu, Z.; Duan, W.; Liu, F. First-principles calculations on the effect of doping and biaxial tensile strain on electron-phonon coupling in graphene. Phys. Rev. Lett. 2013, 111, No. 196802.

(19) Abdelsayed, V.; Moussa, S.; Hassan, H. M.; Aluri, H. S.; Collinson, M. M.; El-Shall, M. S. Photothermal deoxygenation of graphite oxide with laser excitation in solution and graphene-aided increase in water temperature. J. Phys. Chem. Lett. 2010, 1, 28042809.

(20) Kalita, G.; Qi, L.; Namba, Y.; Wakita, K.; Umeno, M. Femtosecond laser induced micropatterning of graphene film. Mater. Lett. 2011, 65, 1569-1572.

(21) Herziger, F.; Mirzayev, R.; Poliani, E.; Maultzsch, J. In-situ Raman study of laser-induced graphene oxidation. Phys. Status Solidi B 2015, 252, 2451-2455.

(22) Krauss, B.; Lohmann, T.; Chae, D.-H.; Haluska, M.; von Klitzing, K.; Smet, J. H. Laser-induced disassembly of a graphene single crystal into a nanocrystalline network. Phys. Rev. B: Condens. Matter Mater. Phys. 2009, 79, No. 165428.

(23) Kiisk, V.; Kahro, T.; Kozlova, J.; Matisen, L.; Alles, H. Nanosecond laser treatment of graphene. Appl. Surf. Sci. 2013, 276, 133-137.

(24) Pittenger, B.; Erina, N.; Su, C., Bruker application note AN128, Rev. B0, 2012.

(25) Pittenger, B.; Erina, N.; Su, C. Mechanical property mapping at the nanoscale using PeakForce QNM scanning probe technique. Nanomechanical Analysis of High Performance Materials; Springer, 2014; pp 31-51.

(26) Johansson, A.; Myllyperkiö, P.; Koskinen, P.; Aumanen, J.; Koivistoinen, J.; Tsai, H.-C.; Chen, C.-H.; Chang, L.-Y.; Hiltunen, V.M.; Manninen, J. J.; et al. Optical Forging of Graphene into ThreeDimensional Shapes. Nano Lett. 2017, 17, 6469-6474.

(27) Aumanen, J.; Johansson, A.; Koivistoinen, J.; Myllyperkiö, P.; Pettersson, M. Patterning and tuning of electrical and optical properties of graphene by laser induced two-photon oxidation. Nanoscale 2015, 7, 2851-2855.

(28) Malard, L.; Pimenta, M.; Dresselhaus, G.; Dresselhaus, M. Raman spectroscopy in graphene. Phys. Rep. 2009, 473, 51-87.

(29) Ferrari, A. C.; Meyer, J.; Scardaci, V.; Casiraghi, C.; Lazzeri, M.; Mauri, F.; Piscanec, S.; Jiang, D.; Novoselov, K.; Roth, S.; et al. Raman spectrum of graphene and graphene layers. Phys. Rev. Lett. 2006, 97, No. 187401.

(30) Lee, J.-U.; Yoon, D.; Cheong, H. Estimation of Young's modulus of graphene by Raman spectroscopy. Nano Lett. 2012, 12, 4444-4448.

(31) Lee, J. E.; Ahn, G.; Shim, J.; Lee, Y. S.; Ryu, S. Optical separation of mechanical strain from charge doping in graphene. Nat. Commun. 2012, 3, No. 1024.

(32) Kip, B. J.; Meier, R. J. Determination of the local temperature at a sample during Raman experiments using Stokes and anti-Stokes Raman bands. Appl. Spectrosc. 1990, 44, 707-711.

(33) Marigheto, N.; Kemsley, E.; Potter, J.; Belton, P.; Wilson, R. Effects of sample heating in FT-Raman spectra of biological materials. Spectrochim. Acta, Part A 1996, 52, 1571-1579.

(34) Lanzi, M.; Di-Nicola, F. P.; Livi, M.; Paganin, L.; Cappelli, F.; Pierini, F. Synthesis and characterization of conjugated polymers for the obtainment of conductive patterns through laser tracing. J. Mater. Sci. 2013, 48, 3877-3893.

(35) Lee, J. H.; Tan, J. Y.; Toh, C.-T.; Koenig, S. P.; Fedorov, V.; Castro Neto, A. H.; Ozyilmaz, B. Nanometer thick elastic graphene engine. Nano Lett. 2014, 14, 2677-2680.

(36) Deng, Z.; Klimov, N. N.; Solares, S. D.; Li, T.; Xu, H.; Cannara, R. J. Nanoscale interfacial friction and adhesion on supported versus suspended monolayer and multilayer graphene. Langmuir 2012, 29, 235-243.

(37) Ryu, S.; Liu, L.; Berciaud, S.; Yu, Y.-J.; Liu, H.; Kim, P.; Flynn, G. W.; Brus, L. E. Atmospheric oxygen binding and hole doping in deformed graphene on a $\mathrm{SiO} 2$ substrate. Nano Lett. 2010, 10, 49444951.

(38) Kazakova, O.; Panchal, V.; Burnett, T. L. Epitaxial graphene and graphene-based devices studied by electrical scanning probe microscopy. Crystals 2013, 3, 191-233.

(39) Melios, C.; Centeno, A.; Zurutuza, A.; Panchal, V.; Giusca, C. E.; Spencer, S.; Silva, S. R. P.; Kazakova, O. Effects of humidity on the electronic properties of graphene prepared by chemical vapour deposition. Carbon 2016, 103, 273-280.

(40) Lee, N.; Yoo, J.; Choi, Y.; Kang, C.; Jeon, D.; Kim, D.; Seo, S.; Chung, $H$. The interlayer screening effect of graphene sheets investigated by Kelvin probe force microscopy. Appl. Phys. Lett. 2009, 95, No. 222107.

(41) Salary, M. M.; Inampudi, S.; Zhang, K.; Tadmor, E. B.; Mosallaei, H. Mechanical actuation of graphene sheets via optically induced forces. Phys. Rev. B: Condens. Matter Mater. Phys. 2016, 94, No. 235403.

(42) Zhang, Y.; Son, H.; Zhang, J.; Kong, J.; Liu, Z. Laser-heating effect on Raman spectra of individual suspended single-walled carbon nanotubes. J. Phys. Chem. C 2007, 111, 1988-1992.

(43) Lazzeri, M.; Mauri, F. Nonadiabatic Kohn anomaly in a doped graphene monolayer. Phys. Rev. Lett. 2006, 97, No. 266407.

(44) Piscanec, S.; Lazzeri, M.; Mauri, F.; Ferrari, A.; Robertson, J. Kohn anomalies and electron-phonon interactions in graphite. Phys. Rev. Lett. 2004, 93, No. 185503.

(45) Beams, R.; Cançado, L. G.; Novotny, L. Raman characterization of defects and dopants in graphene. J. Phys.: Condens. Matter 2015, 27, No. 083002.

(46) Cançado, L. G.; Jorio, A.; Ferreira, E. M.; Stavale, F.; Achete, C.; Capaz, R.; Moutinho, M.; Lombardo, A.; Kulmala, T.; Ferrari, A. Quantifying defects in graphene via Raman spectroscopy at different excitation energies. Nano Lett. 2011, 11, 3190-3196.

(47) Martins, L. G. P.; Matos, M. J.; Paschoal, A. R.; Freire, P. T.; Andrade, N. F.; Aguiar, A. L.; Kong, J.; Neves, B. R.; de Oliveira, A. B.; Mazzoni, M. S. Raman evidence for pressure-induced formation of diamondene. Nat. Commun. 2017, 8, No. 96.

(48) Calizo, I.; Bejenari, I.; Rahman, M.; Liu, G.; Balandin, A. A. Ultraviolet Raman microscopy of single and multilayer graphene. $J$. Appl. Phys. 2009, 106, No. 043509.

(49) Mueller, N. S.; Heeg, S.; Alvarez, M. P.; Kusch, P.; Wasserroth, S.; Clark, N.; Schedin, F.; Parthenios, J.; Papagelis, K.; Galiotis, C.; et al. Evaluating arbitrary strain configurations and doping in graphene with Raman spectroscopy. 2D Mater. 2017, 5, No. 015016.

(50) Mohr, M.; Maultzsch, J.; Thomsen, C. Splitting of the Raman 2 D band of graphene subjected to strain. Phys. Rev. B: Condens. Matter Mater. Phys. 2010, 82, No. 201409.

(51) Bunch, J. S.; Verbridge, S. S.; Alden, J. S.; Van Der Zande, A. M.; Parpia, J. M.; Craighead, H. G.; McEuen, P. L. Impermeable atomic membranes from graphene sheets. Nano Lett. 2008, 8, 24582462 .

(52) Koenig, S. P.; Boddeti, N. G.; Dunn, M. L.; Bunch, J. S. Ultrastrong adhesion of graphene membranes. Nat. Nanotechnol. 2011, 6, 543.

(53) Mackenzie, D. M.; Buron, J. D.; Whelan, P. R.; Jessen, B. S.; Silajdźić, A.; Pesquera, A.; Centeno, A.; Zurutuza, A.; Bøggild, P.; Petersen, D. H. Fabrication of CVD graphene-based devices via laser ablation for wafer-scale characterization. $2 D$ Mater. 2015, 2, No. 045003.

(54) Xing, G.; Guo, H.; Zhang, X.; Sum, T. C.; Huan, C. H. A. The physics of ultrafast saturable absorption in graphene. Opt. Express 2010, 18, 4564-4573.

(55) Currie, M.; Caldwell, J. D.; Bezares, F. J.; Robinson, J.; Anderson, T.; Chun, H.; Tadjer, M. Quantifying pulsed laser induced damage to graphene. Appl. Phys. Lett. 2011, 99, No. 211909. 
(56) Lucchese, M. M.; Stavale, F.; Ferreira, E. M.; Vilani, C.; Moutinho, M.; Capaz, R. B.; Achete, C.; Jorio, A. Quantifying ioninduced defects and Raman relaxation length in graphene. Carbon 2010, 48, 1592-1597.

(57) Eda, G.; Chhowalla, M. Chemically derived graphene oxide: towards large-area thin-film electronics and optoelectronics. Adv. Mater. 2010, 22, 2392-2415.

(58) Oliverio, M.; Perotto, S.; Messina, G. C.; Lovato, L.; De Angelis, F. Chemical functionalization of plasmonic surface biosensors: A tutorial review on issues, strategies, and costs. ACS Appl. Mater. Interfaces 2017, 9, 29394-29411.

(59) Lu, J.; Wu, J.; Carvalho, A.; Ziletti, A.; Liu, H.; Tan, J.; Chen, Y.; Castro Neto, A.; Ozyilmaz, B.; Sow, C. H. Bandgap engineering of phosphorene by laser oxidation toward functional $2 \mathrm{D}$ materials. ACS Nano 2015, 9, 10411-10421.

(60) Zhao, B.; Song, Y.; Wang, S.; Dai, B.; Zhang, L.; Dong, Y.; Lü, J.; Hu, J. Mechanical mapping of nanobubbles by PeakForce atomic force microscopy. Soft Matter 2013, 9, 8837-8843. 


\section{Supplementary Information}

Laser Based Texturing of Graphene to locally tune Electrical Potential and Surface Chemistry

Manoj Tripathi ${ }^{1 *}$, Alice King ${ }^{1}$, Giuseppe Fratta ${ }^{1}$, Manuela Meloni ${ }^{1}$, Matthew Large ${ }^{1}$, Jonathan P. Salvage $^{2}$, Nicola Maria Pugno ${ }^{3,4,5}$, Alan B. Dalton ${ }^{1 * *}$

${ }^{1}$ Department of Physics and Astronomy, University of Sussex, Brighton BN1 9RH, U.K. ${ }^{2}$ School of Pharmacy and Biomolecular Science, University of Brighton, Brighton BN2 4GJ, U.K. ${ }^{3}$ Laboratory of Bio-Inspired \& Graphene Nanomechanics, Department of Civil, Environmental and Mechanical Engineering, University of Trento, Via Mesiano 77, I-38123 Trento, Italy.

${ }^{4}$ School of Engineering and Materials Science, Queen Mary University of London, Mile End Road, E1 4NS London - United Kingdom

${ }^{5}$ Ket Lab, Edoardo Amaldi Foundation, Italian Space Agency, Via del Politecnico snc, I-00133 Roma, Italy

*corresponding author email: m.tripathi@sussex.ac.uk

**co-corresponding author email: a.b.dalton@sussex.ac.uk

(a)
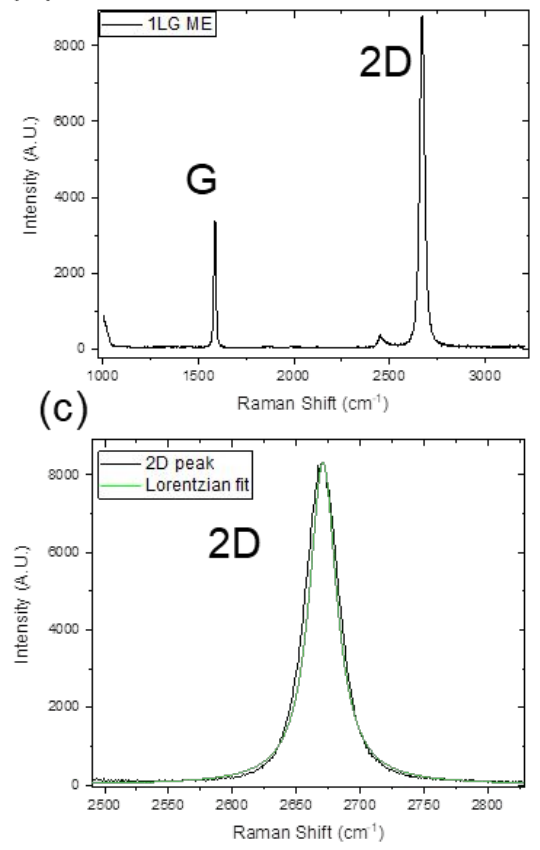

(b)
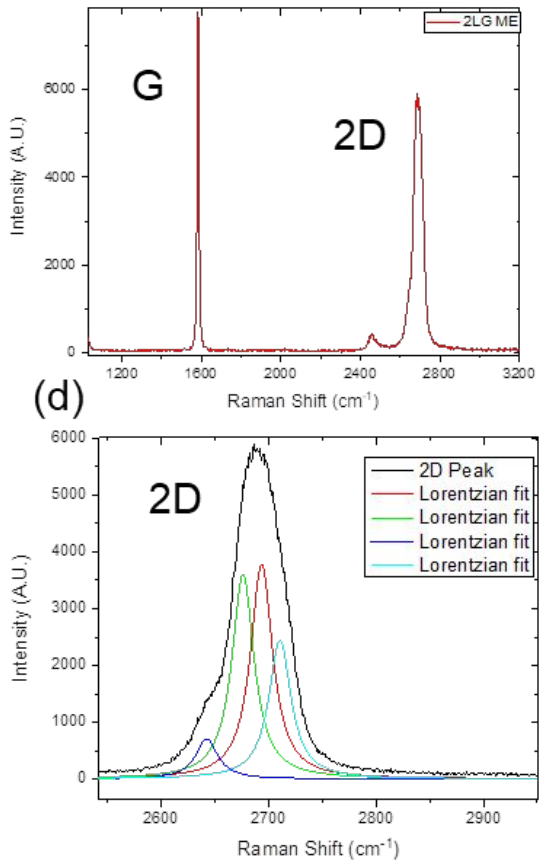
Figure S1: (a) Raman spectra of $1 L G$ and (b) $2 L G$ at lower dose energy showing $\mathrm{G}$ and 2D peaks without any D peaks. The confirmation of the thickness of graphene is carried out through Lorentzian fitting; FWHM $=25 \mathrm{~cm}^{-1}$ for the $1 L G$ in panel (c) and 4 Lorentzian curves for the $2 L G$ in panel (d). The ration between $2 \mathrm{D} / \mathrm{G}$ also indicates presence of $l L G(2 \mathrm{D} / \mathrm{G}>1)$ and $2 L G(2 \mathrm{D} / \mathrm{G}$ $<1)$.
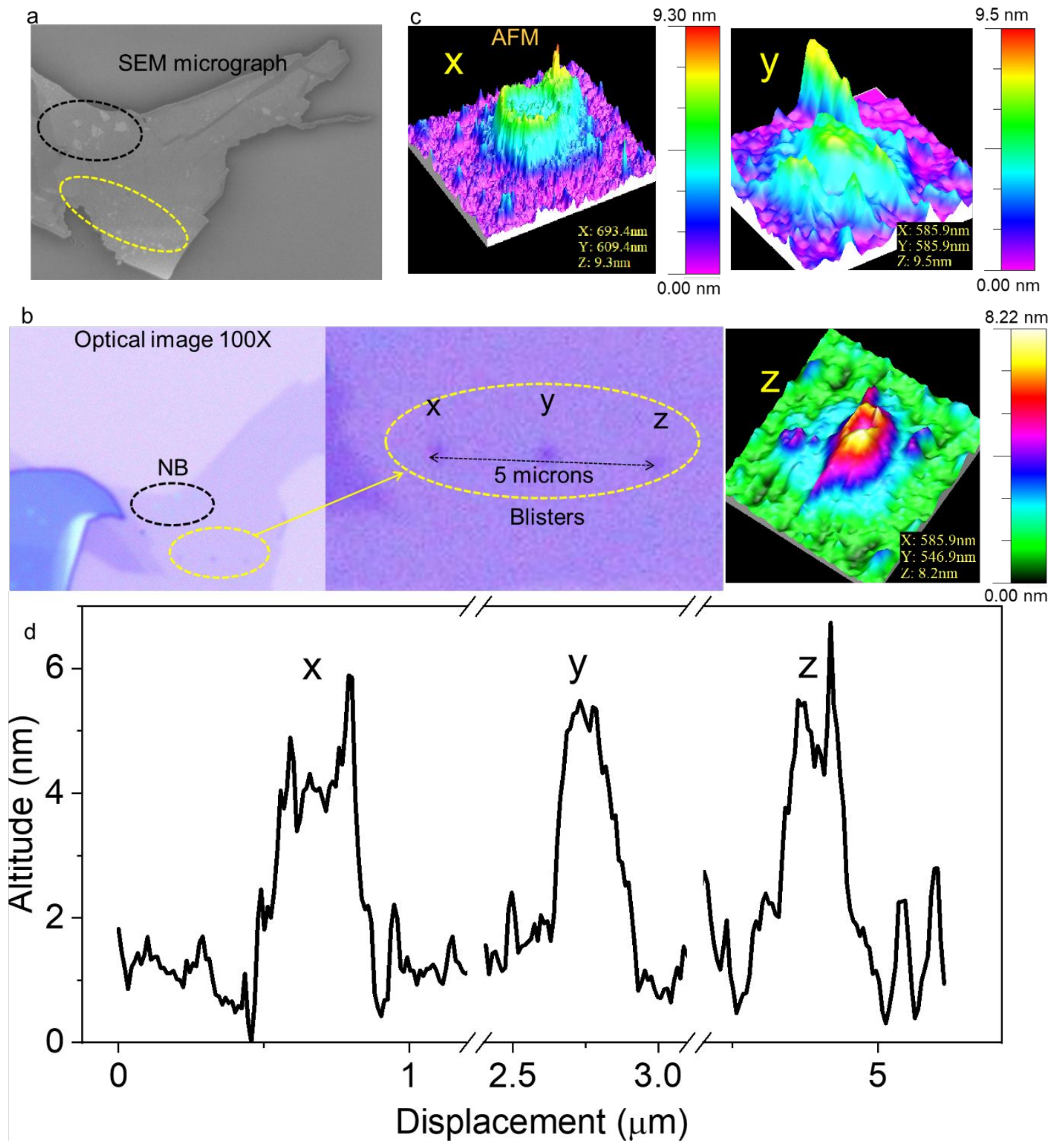
Figure S2: (a) SEM micrograph of an 1LG graphene has graphene nanobubbles (marked by black color dashed oval region) and graphene blisters marked by yellow color oval region. (b) Optical image of the same region showing the presence graphene nanobubbles and blisters produced at laser dose $753 \mathrm{~mJ} / \mu \mathrm{m}^{2}$. Inset shows high resolution optical image of the three blisters labelled as $\mathrm{a}, \mathrm{b}$ and c with separation of 2 microns from each other. (c) AFM image of 3-D topography of each blister showing consistency in the altitude as confirmed by line profile (d).

(a) Topography

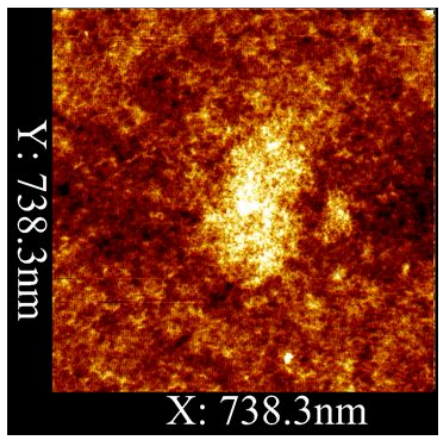

(b)

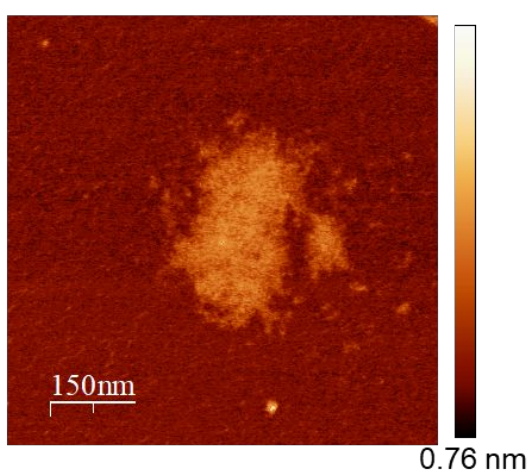

(c) Adhesion map $873 \mathrm{pN}$

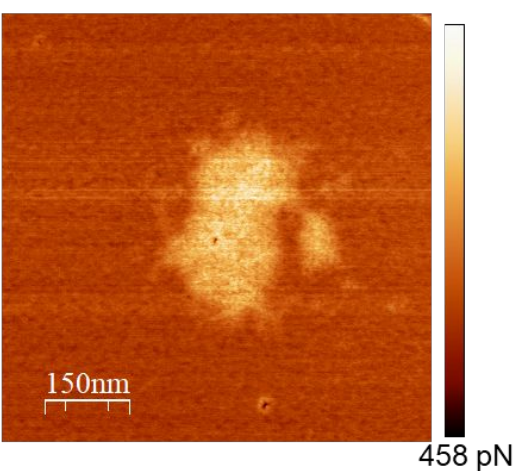

Figure S3: (a) Topography, (b) deformation and (c) adhesion map of individual blister of graphene. The AFM probe senses various interactions at different separation from the substrate. The "peak force" is the maximum deflection of the cantilever and is controlled by the "set-point" to minimise the damage to the tip and the surface (i.e. blister altitude). The maximum deformation of the sample is defined as the separation distance from the base of the "deformation fit region" position to the peak interaction force position. The adhesion map is measured during "pull-out" event by analyzing "backward" curve. The adhesion force is strongly correlated to the deformation of the blister due to increase in contact area between the probe and the graphene carbon atoms during the "forward" approach.

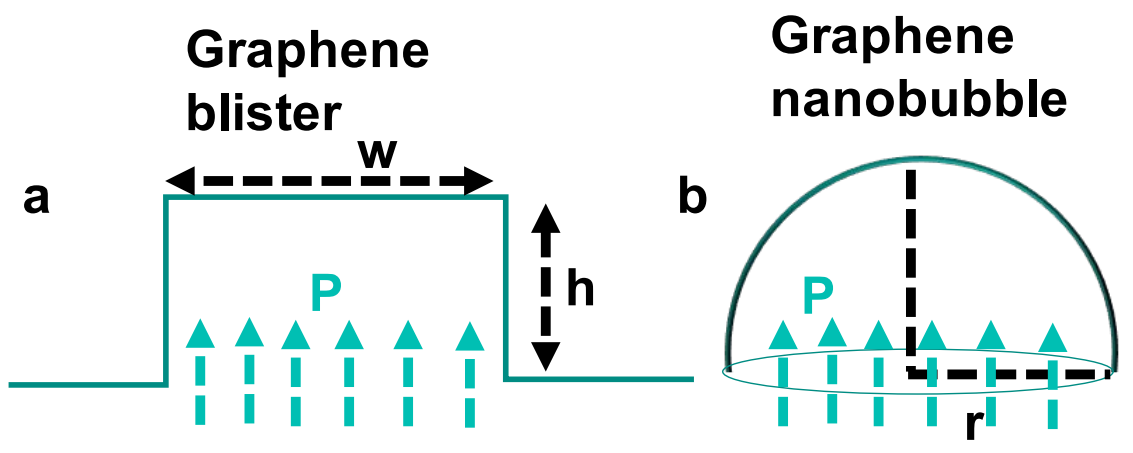


Figure S4: Schematic view illustrating evaluation of the internal pressure in (a) graphene blister and (b) graphene nanobubble.

$$
\begin{aligned}
& P w^{2}=\sigma 4 w t \\
& P \pi r^{2}=\sigma t(2 \pi r)
\end{aligned}
$$

Where, $\sigma($ stress $)=\mathrm{E}($ Young's modulus, $1 \mathrm{TP}) \varepsilon($ strain $), \mathrm{r}($ radii $) \approx \mathrm{w}($ width $) / 2$ and $\mathrm{t}$ is thickness of graphene $(0.35 \mathrm{~nm})$.

$$
P=\frac{4 t E \epsilon}{w}
$$

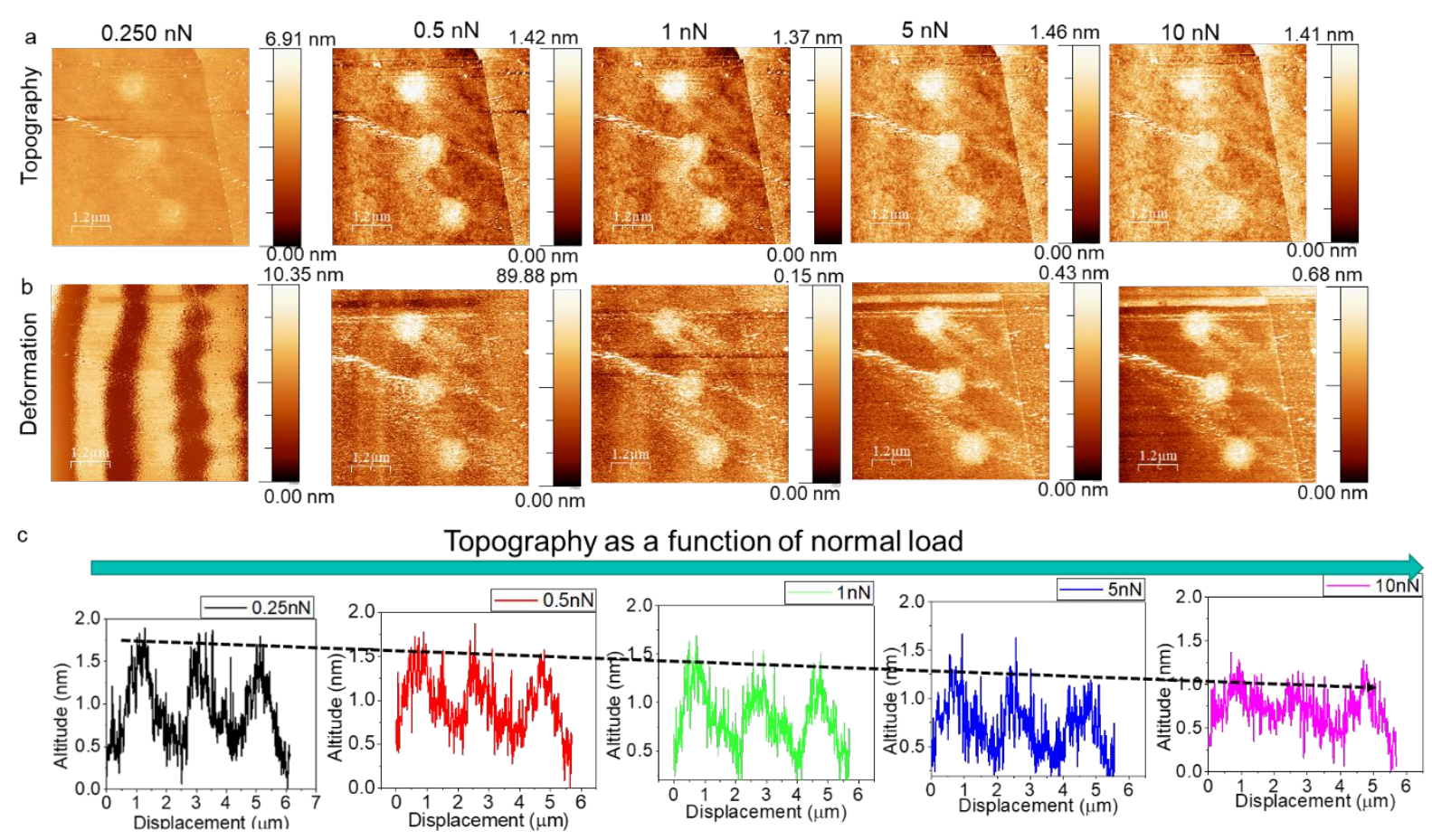

Figure S5: (a) Topography of the blisters of graphene as the function of applied normal force by controlling "set-point" as $0.25 \mathrm{nN}, 0.5 \mathrm{nN} .1 \mathrm{nN}, 5 \mathrm{nN}$ and $10 \mathrm{nN}$ (from left to right panels). The lowest force of $0.25 \mathrm{nN}$ is the threshold of our instrument and electronic noise is dominating in the deformation map. (b) The deformation map represents the pressing of each blisters at different normal force. (c) Line profile at different normal force over three graphene blisters showing 
systematically decreasing of the altitude of blisters. Maximum altitude of the blister is recorded at lowest applied normal force and lowest amplitude is measured at highest normal force of $10 \mathrm{nN}$.
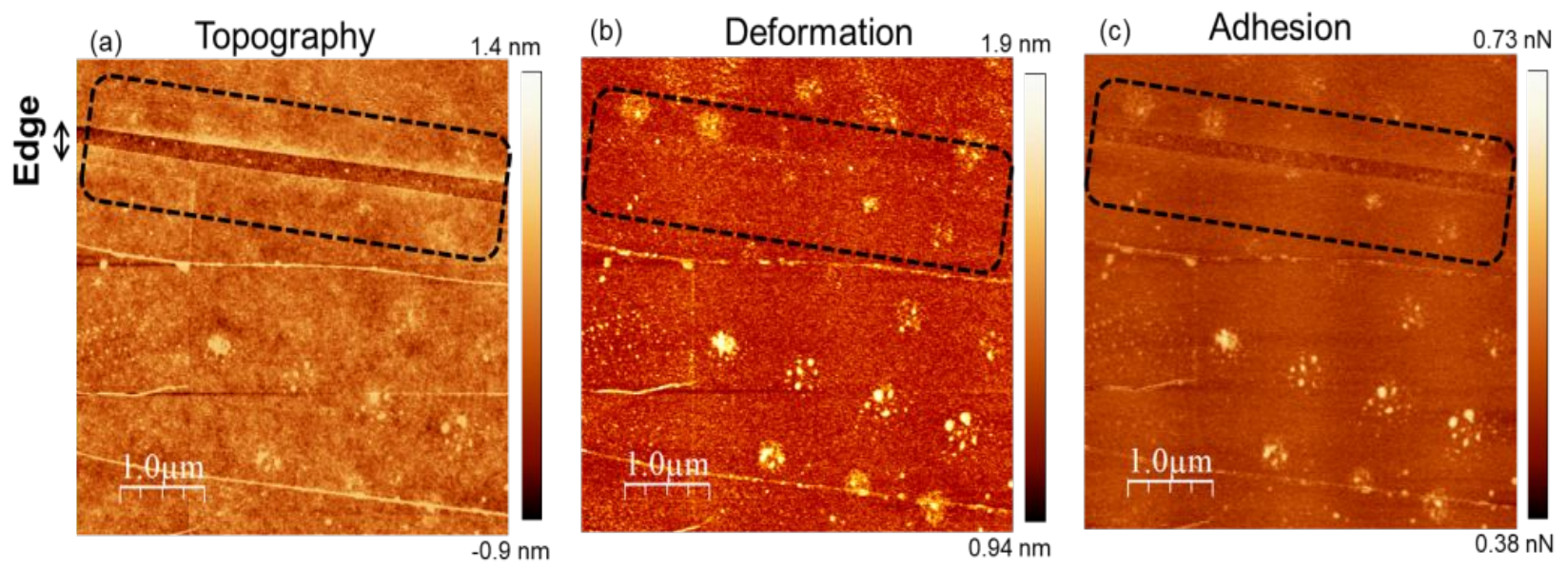

Figure S6: (a) AFM topography of graphene blisters at the graphene edge region. Two single layer of graphene edges are separated by exposed silica substrate. The presence of blistered graphene are evident at the basal plain region away from the edges. The topography signal diminishes near the edge region shows the minimal elevation of the altitude of the graphene blisters. The signal associated with mechanical deformation in panel (b) and adhesion force map in panel (c) shows better contrast which are associated to lattice expension and oxidization. It indicated the role of trapped molecules for the elevation of the graphene blisters.
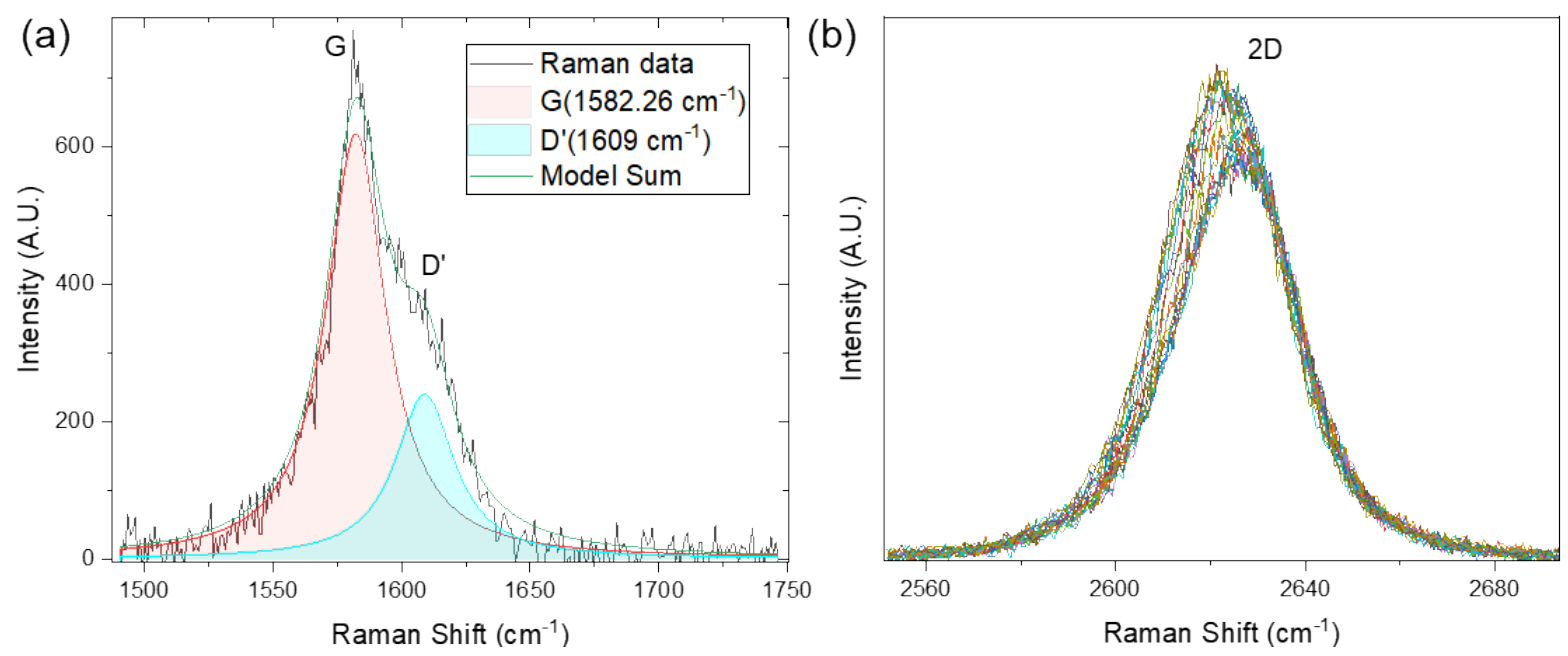
Figure S7: (a) Raman spectrum of G and D' peak measured at the central region of the blister. (b) Spectra of 2D peaks showing systematic redshift as a function of displacement on an individual blister.

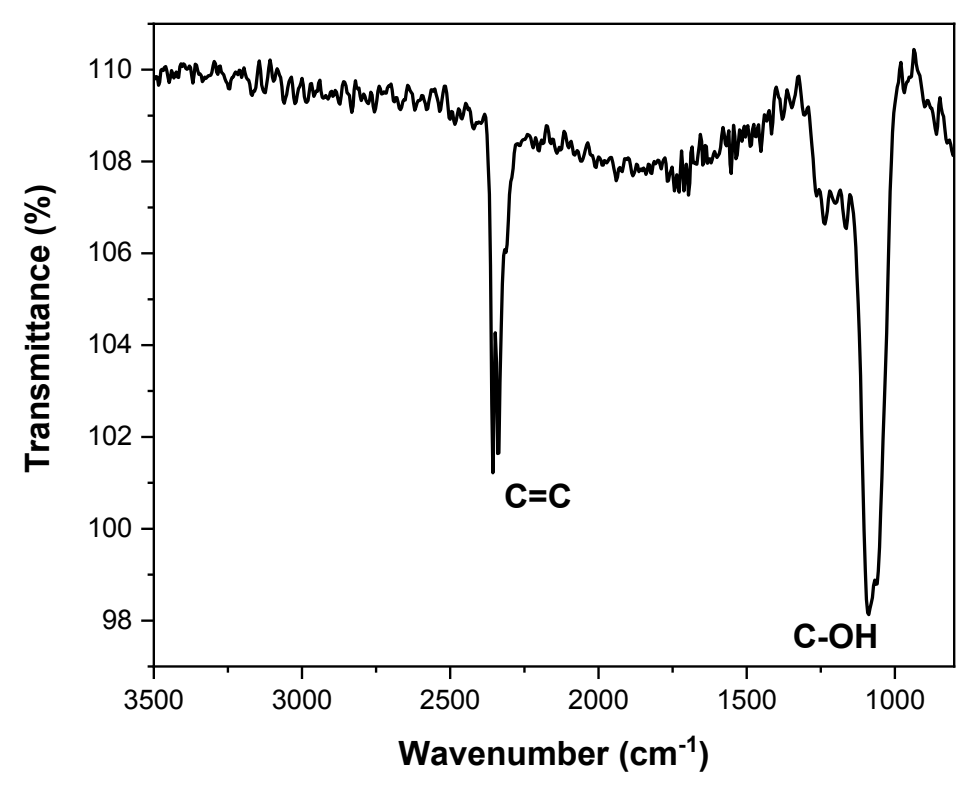

Figure S8: FT-IR spectra of $1 L G$ after laser treatment shows the presence of oxygenic functional groups.
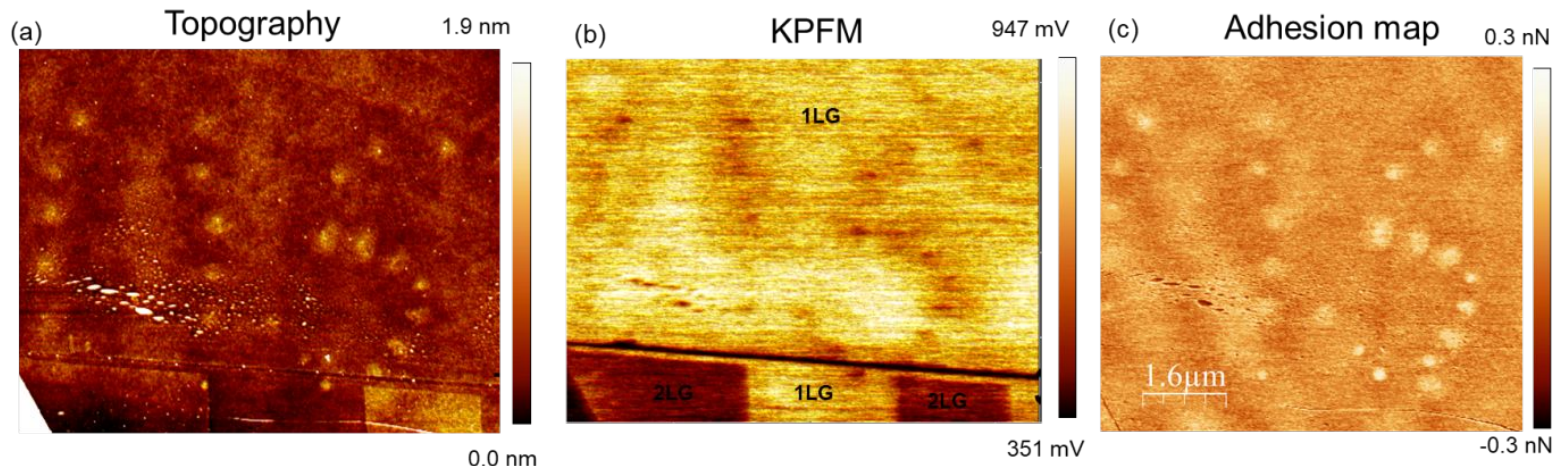

Figure S9: Laser writing induced Tuning of graphene. 3-D blister preparation in the design of US (University of Sussex) with contradistinguish in (a) Topography, (b) CPD and (c) adhesion map. 